\title{
Eine uniforme Analyse sententialer Proformen im Deutschen
}

\begin{abstract}
Der Aufsatz diskutiert die Syntax und Semantik von Konstruktionen im Deutschen mit es- und präpositionalen Korrelaten wie Leo bedauert es, dass Mia krank ist oder Leo freut sich darüber, dass Mia gesund ist. Er argumentiert gegen Breindls (1989), Sudhoffs (2003, i.Vorb.), und Freys (2011) Homonymiehypothese, nach der es-Korrelate und präpositionale Korrelate sich jeweils in unterschiedliche syntaktische Kategorien aufsplitten. Es wird eine uniforme Analyse präsentiert, die generell ein Korrelat als eine Proform ansieht, die auf ein abstraktes Objekt referiert. Letzteres ist entweder eine Aussage $\sigma$, auf die ein eingebetteter Deklarativsatz oder das Radikal eines eingebetteten wenn- oder $o b$-Satzes referiert, oder es handelt sich bei ihm um eine kontextgegebene Aussage $\mu$, die eine Antwort auf die eingebettete $w$-Frage darstellt. Die uniforme Analyse offeriert syntaktische und semantische Erklärungen für die Beobachtungen, die zu der Homonymiehypothese geführt haben.

The paper discusses the syntax and semantics of German constructions with es- and prepositional correlates like Leo bedauert es, dass Mia krank ist 'Leo regrets it that Mia is ill' and Leo freut sich darüber, dass Mia krank ist 'Leo is happy $d a$-about that Mia is ill'. It argues against Breindl's (1989), Sudhoff's (2003), and Frey's (2011) homonymy accounts that distinguish between different syntactical correlate categories. It presents a uniform analysis where a correlate is generally regarded as a proform that refers to an abstract object. The latter is a statement $\sigma$ which either is referred to by an embedded declarative or the radical of an embedded wenn- or $o b$-clause or it is a contextually given answer $\mu$ to an embedded $w h$-interrogative. The uniform analysis offers syntactic and semantic explanations for observations that have given rise to the homonymy accounts.
\end{abstract}

\section{Einleitung}

Unter einem Korrelat versteht man im Allgemeinen eine Proform im Matrixsatz, die sich in bestimmter Weise auf einen subordinierten Satz, ihren Bezugssatz, bezieht. In der Literatur werden sie als „Platzhalter“, ,anaphorische Proformen“ oder als „Bezugselemente“ bezeichnet, wenn sie durch ihren Bezugssatz substituierbar sind - vgl. (1a-c) sowie Reis (1997), Breindl (1989), Zifonun/Hoffmann/Strecker (1997) und Sudhoff (2003). Wir zählen zu den Korrelaten auch Proformen in Konstruktionen mit ergänzendem wenn-Satz wie in (1d). Diese beziehen sich zwar auch auf den subordinierten Satz, sind aber durch diesen nicht ersetzbar - vgl. Fabricius-Hansen (1980), Kaiaty (2010) und Schwabe (i.Vorb.).

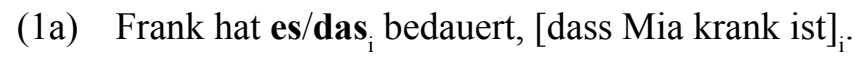

(1b) Frank hat sich darüber ${ }_{\mathrm{i}}$ gefreut, [dass Mia wieder gesund ist $]_{\mathrm{i}}$.

(1c) Frank freut sich dann ${ }_{\mathrm{i}}$, [wenn Mia gesund ist $]_{\mathrm{i}}$.

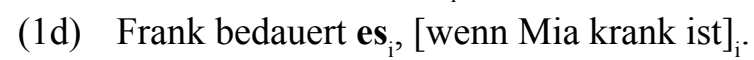

Dieser Aufsatz behandelt Korrelate zu Argumentausdrücken. Das heißt, Korrelate zu Supplementen wie in (1c) werden nur am Rand betrachtet. Es wird diskutiert, wovon die Wahl eines Korrelats als es beziehungsweise das oder als präpositionales Korrelat (ProPP) abhängt, in welcher Beziehung es zu seinem Bezugssatz steht und welche syntaktische und gegebenenfalls semantische Funktion es hat. Gezeigt werden soll, dass bei es-Korrelaten und ProPPn die empirisch motivierte Unterscheidung von Platzhaltern einerseits und 
Proformen beziehungsweise Bezugselementen andererseits nicht zwingend ist. Korrelate werden immer als Proformen analysiert, die auf eine abstrakte Aussage referieren.

Der syntaktischen und semantischen Analyse ist ein empirischer Überblick vorangestellt, der zeigt, mit welchen syntaktischen Funktionen Korrelate korrelieren, welchen Satztyp ihre Bezugssätze haben können, und inwieweit sie obligatorisch, optional oder gar ausgeschlossen sind. Es handelt sich bei den Daten um Korpusbelege aus der ZAS-Datenbank zu satzeinbettenden Prädikaten. Letztere enthält etwa 1700 satzeinbettende Prädikate.

\section{Korrelatsyntax}

\subsection{Syntaktische Verbklassen und Korrelate}

Ein nominales Argument, das zu einem Verb in einer Argumentbeziehung steht beziehungsweise im Verbalkomplex (vP) basisgeneriert ist, steht in einer besonders engen Relation zum Matrixverb. Huddleston/Pullum (2002) bezeichnen es deswegen auch als „,core complement". Befindet sich so ein Kernkomplement innerhalb des engeren Verbalkomplexes (VP), ist es ein internes Argument und fungiert als direktes Objekt. Figuriert es außerhalb der VP, ist es ein externes Argument und erfüllt die Funktion eines Subjekts. Nominale Komplemente in diesen Positionen werden typischerweise im Nominativ beziehungsweise Akkusativ realisiert. Gibt es ein weiteres internes Argument, erscheint dieses im Dativ und fungiert als indirektes Objekt. Außer den direkten und indirekten Objekten können innerhalb der VP noch weitere nominale Komplemente erscheinen. Sie sind dann als Genitiv realisiert oder über eine Präposition, also indirekt, mit dem verbalen Kopf verbunden. Die Präposition ist der Kopf einer Präpositionalphrase (PP), der das nominale Komplement regiert. Die PP ihrerseits wird von einem verbalen Kopf, dem Matrixprädikat, regiert. Komplemente, die über eine Präposition mit dem verbalen Kopf verbunden sind oder als Genitiv realisiert werden, werden auch als oblique Komplemente bezeichnet.

Argumentsätze können all die eben genannten Argumentfunktionen erfüllen. Sie können, je nach Matrixprädikat, als Kernkomplemente oder oblique Komplemente vorkommen. Die Art der Verknüpfung mit ihrem verbalen Kopf kann durch ein Korrelat signalisiert werden. Neben dieser grammatischen Funktion erfüllt ein Korrelat eine semantische, nämlich auf die Aussage zu referieren, die durch den Bezugssatz bezeichnet wird - vgl. Bennis (2004) und Haider (2010, S. 218). Dass ein Korrelat außerdem die Bedeutung des Matrixprädikats modifizieren kann, werden wir in Abschnitt 3 sehen. Wie sein Bezugssatz kann ein Korrelat ein Komplement eines Matrixprädikats sein (1a, b, d) oder auch ein Modifikator (1c). Als Komplement steht es entweder zu einem Matrixverb oder zu einer Präposition in einer Kopfbeziehung. Ist ersteres der Fall, handelt es sich um ein es- oder das-Korrelat. Beide markieren die Subjekt- beziehungsweise direkte Objektfunktion. ${ }^{1}$ Ist das Korrelat in eine Präposition integriert, handelt es sich um eine ProPP wie darin, damit, davor, deswegen - vgl. (1b). Korrelate können Deklarativ-, Interrogativ-, Infinitiv-, Adverbial- und marginal V2-Sätze als Bezugssätze haben - vgl. (2a-c) und (9a-c'). ${ }^{2}$ Schauen wir uns die Prädikatsklassen mit $i$. Objekt-es, ii. Subjekt-es und iii. ProPP im Einzelnen an.

In der ZAS-Datenbank sind zwei Verben mit Dativkorrelat belegt (zustimmen und widersprechen) und 26 Verben mit Genitivkorrelat (z.B. belehren und entsinnen).

2 Von den 1700 satzeinbettenden Prädikaten der ZAS-Datenbank selegieren 49\% nur dass-Sätze (folgern, glauben , ...), 28\% dass- oder ob- oder wh-Sätze (wissen, bedenken, ...), 19\% dass- oder wh-Sätze (bedauern, sich freuen, ...), 3\% dass- oder ob-Sätze (absichern, dementieren, ...) und 1\%ob-oder wh-Sätze. 


\subsubsection{Verben mit Objekt-es}

Die knapp 800 Verben mit einem Objekt-es subkategorisieren, wie oben bemerkt, einen Komplementsatz, der einem direkten Objekt entspricht - vgl. die Beispiele in (2a-c). Dieses ist meistens obligatorisch - vgl. etwas bedauern vs. (etwas) lesen. Für etwa die Hälfte dieser Verben ist ein Objekt-es belegt.

(2a) Ein wenig bedauert (es) Stalla, dass er seinen Österreichurlaub schon vor Monaten gebucht hat. (DWDS BZ 2004)

(2b) Wir stellen (es) den Dozenten frei, ob sie davon Gebrauch machen möchten. (DWDS Zeit 2007)

(2c) Und dann erzählte er (es), wie er sich gefühlt habe, als er zur Seite sank. (IDS brz 2009)

Es zeigt sich, dass von etwa 400 V2-Einbettern mit Akkusativ nur für etwa vier Prozent ein Satz mit einem Objekt-es exemplifiziert ist - vgl. (3a-c). Bis auf (3a) handelt es sich bei diesen Sätzen um Doppelpunktkonstruktionen oder der eingebettete V2-Satz ist im Konjunktiv - vgl. (3b, c). Dieser Befund ist nicht verwunderlich, denn V2 wird eigentlich durch es-Korrelate blockiert.

(3a) Man sah es ihm an, er wusste sich als einer von Vielen. (DWDS TS 2002)

(3b) Auch wenn es viele nicht begreifen wollen: Eine soziale Marktwirtschaft kann nur so sozial sein, wie sie zuvor effektiv ist. (DWDS BZ 2005)

(3c) Manchmal zöge man es vor, sie dächte mehr an sich. (DWDS Zeit 2004)

Prädikate, die nur $o b$ - und $w$-Komplementsätze einbetten, kommen nicht mit es-Korrelaten vor - vgl. abfragen, anfragen, dazwischenfragen, fragen, herumfragen, nachfragen, untersuchen und rückfragen. Zu diesen Prädikaten gehören auch Verben wie argwöhnen, bei denen es sich um modifikatorische frageeinbettende Prädikate handelt.

(4a) Sie fragen (*es) den Besitzer, ob sie sein Geschäft fotografieren sollen. (DWDS BZ 1994)

(4b) [...] die Gesundheitsbehörden müssen (*es) stets argwöhnen, ob sich eine neue Epidemie anbahnt. (DWDS TS 2003)

Dass der Komplementsatz bei diesen Prädikaten ein direktes Objekt ist, erkennt man daran, dass er mit dem Fragewort was erfragbar oder durch das substituierbar ist. Bei bestimmten deklarativeinbettenden Verben sind es-Korrelate ebenfalls ausgeschlossen. Zu ihnen gehören grölen, kreischen und brummen, die die Art der Äußerung des eingebetteten Satzes denotieren - vgl. (5a), und einschränken in (5b), das den Zweck der Frageäußerung spezifiziert.

(5a) Mein alter Freund Holger. Ich zischte (*es), dass er nie mehr um diese Zeit bei mir klingeln soll. (DWDS BZ 2002)

(5b) Er schränkte (*es) aber ein, nicht über sämtliche Kontakte im politischen und wirtschaftlichen Leben Bescheid zu wissen. (IDS dpr 1998)

Diese Verben lizensieren kein es-Korrelat, weil der eingebettete Satz (noch) nicht zu der lexikalisch determinierten Argumentstruktur des Matrixprädikats gehört.

\subsubsection{Verben mit Subjekt-es}

Für 75 Prozent der Verben, die Subjektsätze lizensieren, ist ein es belegt - vgl. (6a-c). Hier ist aber zu beachten, dass es sich bei diesem häufig nicht um ein es-Korrelat, sondern eher um ein positionales es handelt. Wir kommen in Abschnitt 2.3 darauf zurück. 
(6a) $\mathrm{Zu}$ Beginn der Woche sickerte es durch, dass die Telekom womöglich die Online-Firma Club Internet kauft, [...] (DWDS Zeit 2000)

(6b) Umso mehr erstaunt es, wieviel Anteilnahme [...] sie [...] erfuhr. (DWDS Zeit 1992)

(6c) Es fällt auf, sie alle mahnen [...] vor dem Unheil, [...]. (DWDS Zeit 1983)

\subsubsection{Verben mit ProPP}

Bei den etwa 800 Verben, die ProPPn erlauben, ist bei zwei Dritteln das Aussagenargument obligatorisch - vgl. dazu auffordern und daran liegen. Fakultative Argumente haben beispielsweise sich (darüber) beschweren, (dazu) aufstacheln, (darüber) nachdenken und (darunter) leiden. Bei beiden Verbgruppen gibt es welche mit obligatorischer und welche mit optionaler ProPP - vgl. dafür büßen und (darauf) insistieren in (7) für obligatorische und darüber nachdenken dass und sich (dafür) anstrengen in (8) für fakultative Aussagenargumente. Optional ist die ProPP bei 60 Prozent aller ProPP-Verben - vgl. (9a'-c').

(7a) Jetzt büßen die USA *(dafür), dass sie Militärdiktaturen im Süden gestützt und diesen Teil der Halbinsel als Kolonie behandelt haben. (DWDS BZ 2000)

(7b) Bahnchef Hartmut Mehdorn insistiert (darauf), dass man Netz und Betrieb nicht trennen kann. (DWDS Zeit 2001)

(8a) Wir müssen aber auch *(darüber) nachdenken, dass Bund und Länder bestimmte Aufgaben in Projekten organisieren, um Schwerpunkte zu bilden. (DWDS BZ 2004)

(8b) Niemand strengt sich (dafür) an, dass es ihm hinterher schlechter geht. (DWDS Zeit 2004)

(8b') Wir strengen uns wirklich an, dass wir was einsparen. (DWDS BZ 2001)

Wie bei den Bezugssätzen von es-Korrelaten kommen auch bei denen von ProPPn alle Satztypen vor.

(9a) [...], viele von ihnen konzentrieren sich *(darauf), ob [...] es im Bett noch geht. (DWDS TS 2001)

(9a') Stölzl sollte (darüber) nachdenken, ob er sein Amt als Vizepräsident mit seiner Fraktionsarbeit vereinbaren kann. (DWDS TS 2004)

(9b) Wenn man *(davon) ausgeht, wer sich zuvor durch finstere Miene verdächtig gemacht hat, könnte jeder im Schloss der Mörder gewesen sein. (DWDS TS 2003)

(9b') Man muss auch einmal (darüber) nachdenken, wer wie viel in die Rentenkassen eingezahlt hat. (DWDS TS 2003)

(9c) Ich [...] stehe *(dazu), wir sind 1998 nicht abgewählt worden, weil die Sozialdemokraten so gut waren, sondern [...] (DWDS Zeit 2001)

(9c') Klinge verteidigt sich (damit), er habe doch nichts verschwiegen, [...] (DWDS K-Ge 1957)

Bei etwa 18 Prozent, der ProPP lizensierenden Verben hängt die Optionalität beziehungsweise die Obligatheit der ProPP vom eingebetteten Satztyp ab. Zum Beispiel haben nachdenken, nachgrübeln, nachsinnen und rätseln bei Deklarativeinbettung eine obligatorische und bei Frageeinbettung eine fakultative ProPP - vgl. (9a') und (10).

(10) Unter anderem hatte er laut*(darüber) nachgedacht, dass er wohl die falschen Spieler verpflichtet habe. (DWDS BZ 2000)

Für drei Prozent der ProPP-Verben sind indikativische V2-Einbettungen ohne Doppelpunktlesart exemplifiziert - vgl. (9c). Bei den einebetteten konjunktivischen V2-Sätzen handelt es sich meistens um indirekte oder interne Rede - vgl. (9c'). 
Bei Prädikaten mit obligatorischem Aussagenargument kommen mehr obligatorische ProPPn vor als optionale. Bei Verben mit optionalem Aussagenargument sind optionale ProPPn in der Überzahl. Breindl (1989, S. 189ff.) weist darauf hin, dass sowohl das Aussagenargument als auch die ProPP bei „relationalen Verben“ obligatorisch sind. Zu diesen Verben gehören direktionale Verben wie darauf abzielen und darauf hinweisen und lokale wie davon abhängen und daran liegen. Sie stellen eine direkte Relation zwischen zwei Aussagen beziehungsweise einem Individuum und einer Aussage her. Bei Verben mit optionaler ProPP wie sich (darüber) amüsieren und (darüber) debattieren hingegen ist die Relation zwischen dem Subjekt und der Aussage eher vermittelt. ${ }^{3}$

Bei einer relativ kleinen Klasse von Verben, bei zwei Prozent, variiert bei gleicher Argumentstruktur die Argumentrealisierung. Sie lizensieren sowohl es-Korrelate als auch ProPPn. Zu ihnen gehören zum Beispiel bestimmen, diskutieren, entscheiden, erzählen, glauben, hören, hoffen, raten und wissen.

(11a) Sie spielte es wunderbar, und sie hat es geglaubt, dass es wahr war, aber es war nicht die Wirklichkeit. (DWDS BZ 2004)

(11b) Wir glauben daran, dass Berlin in den kommenden Jahren der Wachstumsmarkt in der Medienbranche sein wird. (DWDS BZ 2004)

\subsection{Analyseansätze für Konstruktionen mit es-Korrelaten}

Hier wird dafür argumentiert, dass Korrelate eine Lexikoneinheit darstellen, eine Proform, die sich auf eine im Kontext gegebene abstrakte Aussage bezieht. Dieser uniforme Ansatz unterscheidet sich von Ansätzen, die es-Korrelate und ProPPn jeweils als Homonyme ansehen. Im Hinblick auf das es-Korrelat, wird zwischen folgenden Varianten unterschie-

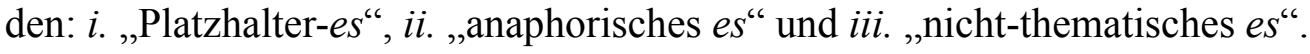

\subsection{1 „Platzhalter-es“}

Pütz (1986), Sudhoff (2003, i.Vorb.) und Frey (2011) unterscheiden zwischen Platzhalteres - Sudhoff nennt es Korrelat-es - und anaphorischem es-Korrelat. Bei letzterem handelt es sich um eine Proform, die auf eine kontextgegebene Aussage referiert. Diese Unterscheidung fußt vor allem darauf, dass bei bedauern-Verben das Objekt-es im Fokus sein kann, bei behaupten-Verben hingegen nicht. In der Theorie von Schwarzschild (1999), nach der eine Konstituente F-markiert ist, wenn sie nicht gegeben ist, kann bei bedauernVerben das Objekt-es F-markiert sein, bei behaupten-Verben hingegen nicht:

(12) Was gibt es Neues?

(12a) ${ }_{F}$ Max bedauert $_{\mathrm{F}} \mathrm{es}_{\mathrm{F}}\left[\right.$ $_{\mathrm{F}}$ dass Mia KRANK $_{\mathrm{F}}$ ist $\left.]\right]$

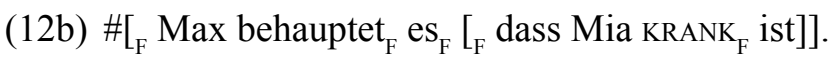

Diese Beobachtung ist nachvollziehbar und wird auch durch psycholinguistische Experimente und Korpusanalysen von Trompelt/Axel-Tober/Holler (i.Vorb.) gestützt. Aus der ZAS-Datenbank geht hervor, dass knapp die Hälfte aller Akkusativ einbettender Verben ein nicht-anaphorisches es-Korrelat erlauben - vgl. Schwabe (2012). Sudhoff (2003) weist

\footnotetext{
3 Die Frage, was die Optionalität beziehungsweise die Obligatheit einer ProPP bedingt, ist spannend, muss aber leider an dieser Stelle offen bleiben.
} 
außerdem darauf hin, dass die behaupten-Verben sowohl $w$-Extraktion als auch V2-Einbettung lizensieren und dass beides durch ein es-Korrelat blockiert wird - vgl. (13a, b). ${ }^{4}$

(13a) Max glaubt (*es), Mia ist nach Paris gefahren.

(13b) Wohin glaubt (*es) Max, dass Mia gefahren ist?

Die Befunde in (12) und (13) versuchen die oben genannten Autoren, mit der kategorialen Unterscheidung anaphorisches versus Platzhalter-es und der damit einhergehenden strukturellen Positionierung des Bezugssatzes zu erklären.

Sudhoff (2003, i.Vorb.) betrachtet das Platzhalter-es als Determinierer einer komplexen Determinierphase (DP) und den Bezugssatz (CP')als dessen Komplement - vgl. (14a). Müller (1995) - für ihn spielt der Unterschied anaphorisches und nicht-anaphorisches Objekt-es keine Rolle - betrachtet das es-Korrelat als nominalen Kopf einer Nominalphrase (NP)und den Komplementsatz als dessen Komplement in Analogie zu einer Struktur wie $\left[_{\mathrm{NP}}\right.$ die $\left[_{\mathrm{N} 0}\right.$ Tatsache $\left.] \mathrm{CP}^{\prime}\right]$ - vgl. (14b).

(14) Max hat es bedauert, dass Mia krank ist.

(14a) $\ldots\left\{\left\{_{\mathrm{DP}}[\mathrm{D} 0 \mathrm{es}] \mathrm{CP}^{\prime}\right\} \ldots\right.$

(14b) $\ldots\left\{\left\{_{\mathrm{NP}}\left[\mathrm{N}_{\mathrm{N} 0}\right.\right.\right.$ es $\left.] \mathrm{CP}^{\prime}\right\} \ldots$

Aufgrund seiner fehlenden Akzentuierbarkeit kann das Platzhalter-es laut Sudhoff (2003, S. 94) kein phonologischer Kopf sein - vgl. (15a). Dieses Unvermögen erzwingt entweder die Extraposition des Bezugssatzes (15b), ein „nicht overtes“ es (15c) oder die Substitution von es durch das (15d).

(15a) $*\left[_{\mathrm{CP}}\right.$ Max hat ... [ ${ }_{\mathrm{V}},\left\{_{\mathrm{DP}}\left[{ }_{\mathrm{D} 0}\right.\right.$ es $]$ dass Mia krank ist $\}$ bedauert $\left.]\right]$

(15b) $\left[_{\mathrm{CP}}\right.$ Max hat $\ldots\left[_{\mathrm{V}},\left\{_{\mathrm{DP}}\left[\mathrm{D}_{\mathrm{D} 0}\right.\right.\right.$ es $\left.] \mathrm{t}_{\mathrm{i}}\right\}$ bedauert $]$ dass Mia krank ist $\left.\mathrm{i}_{\mathrm{i}}\right]$

(15c) $\left[{ }_{\mathrm{CP}}\right.$ Max hat ... $\left[_{\mathrm{V}},\left\{_{\mathrm{DP}}\left[\left[_{\mathrm{D} 0} \varnothing\right]\right.\right.\right.$ dass Mia krank ist $\}$ bedauert $\left.]\right]$

(15d) $\left[_{\mathrm{CP}}\right.$ Max hat ... $\left[\left[_{\mathrm{V}},\left\{_{\mathrm{DP}}\left[_{\mathrm{D} 0}\right.\right.\right.\right.$ das $]$ dass Mia krank ist $\}$ bedauert $\left.]\right]$

Die CP' in (14a) kann, so Sudhoff (2003) und Müller (1995), aus der NP beziehungsweise DP heraus wie in (15b) extraponiert werden. Die Topikalisierung aus der DP beziehungsweise NP ist hingegen nicht möglich - vgl. (16a). Letzteres würde zu einer Inselverletzung führen. Eine Topikalisierung der CP' aus der Extraposition wie in (16b) ist ebenfalls nicht möglich.

(16a) $*\left[_{\mathrm{CP}}[\text { dass Mia krank ist }]_{\mathrm{i}}\right.$ hat Max ... $\left[_{\mathrm{V}},\left\{_{\mathrm{DP}}\left[{ }_{\mathrm{D} 0} \mathrm{es}\right] \mathrm{t}_{\mathrm{i}}\right\}\right.$ bedauert $]$

(16b) $*\left[_{\mathrm{CP}} \text { [dass Mia krank ist }\right]_{\mathrm{i}}$ hat Max ... $\left[_{\mathrm{VP}}\left[{ }_{\mathrm{VP}}\left[{ }_{\mathrm{V}},\left\{_{\mathrm{DP}}\left[{ }_{\mathrm{D} 0} \mathrm{es}\right] \mathrm{t}_{\mathrm{i}}\right\}\right.\right.\right.$ bedauert $\left.\left.\left.]\right] \mathrm{t}_{\mathrm{i}}\right]\right]$

Der Grund für (16b) ist, dass gegensätzliche Bewegung (improper movement), das heißt Bewegung erst nach rechts zur Extraposition und dann nach links zur Topik-Position, verboten ist - vgl. das Principle of Unambiguous Binding (PUB) in (Müller/Sternefeld (1993) sowie Müller (1995) und Sternefeld (2006).

Ist das Platzhalter-es wie in (15c) phonologisch leer, kann der Bezugssatz laut Sudhoff und Sternefeld, wie bei einer es-haltigen Struktur, extraponiert, aber auch zusammen mit dem nicht realisierten DP-Kopf topikalisiert werden - vgl. (17a, b).

(17a) $\left[_{\mathrm{CP}}\right.$ Max hat ... $\left[_{\mathrm{V}},\left\{_{\mathrm{DP}}\left[_{\mathrm{D} 0} \varnothing\right] \mathrm{t}_{\mathrm{i}}\right\}\right.$ bedauert $]$ dass Mia krank ist $\left.\mathrm{i}_{\mathrm{i}}\right]$

(17b) $\left[_{\mathrm{CP}}\left\{\left\{_{\mathrm{DP}}\left[{ }_{\mathrm{D} 0} \varnothing\right] \text { dass Mia krank ist }\right\}_{\mathrm{i}}\right.\right.$ Max hat ... $\left[_{\mathrm{V}}, \mathrm{t}_{\mathrm{i}}\right.$ bedauert $\left.]\right]$

4 Dass ein es-Korrelat V2-Einbettung nicht generell blockiert, wurde oben in (7) gezeigt und wird auch, wie wir unten sehen werden, beim Subjekt-es deutlich. 
Hier ergibt sich nun eine Unstimmigkeit. Konstruktionen mit komplexen PPn wie (18a, b) oder Phrasen mit einem dass-Kopf wie (18c) zeigen, dass der pronominale Teil des Korrelats immer prominent akzentuiert ist, so dass das Korrelat gar nicht getilgt werden kann - vgl. (18d, e). Autoren wie Sudhoff müssten also erklären, warum das nicht akzentuierbare es-Korrelat überhaupt als Kopf in Betracht kommt.

(18a) $\left[_{\mathrm{CP}}\right.$ Max hat sich ... $\left[_{\mathrm{V}}\right.$, $\{$ PP DArüber [dass Mia gesund ist $\left.]\right\}$ gefreut $\left.]\right]$

(18b) $\left[_{\mathrm{CP}}\left\{{ }_{\mathrm{PP}}\right.\right.$ DArüber [dass Mia gesund ist] $\}$ i hat sich Max ... $\left[_{\mathrm{V}}, \mathrm{t}_{\mathrm{I}}\right.$ gefreut]

(18c) $\left[_{\mathrm{CP}} \text { Max hat ... [ }{ }_{\mathrm{V}},\left\{_{\mathrm{DP}} \text { DAs [dass Mia krank ist }\right]\right\}_{1}$ geahnt $\left.]\right]$

(18d) $*\left[_{C P} \text { Max hat sich ... [ }\left\{_{P P} \varnothing \text { [dass Mia gesund ist }\right]\right\}_{\mathrm{i}} \mathrm{t}_{\mathrm{i}}$ gefreut $\left.]\right]$

$(18 \mathrm{e}) *\left[_{\mathrm{CP}}\left\{_{\mathrm{PP}} \varnothing[\text { dass Mia gesund ist }]\right\}_{\mathrm{i}}\right.$ hat sich Max $\ldots\left[_{\mathrm{V}}, \mathrm{t}_{\mathrm{i}}\right.$ gefreut $\left.]\right]$

Ein weiterer Kritikpunkt ist der, dass die phonologische Tilgung des es-Korrelats nicht seinem semantischen Verhalten Rechnung trägt. Darauf dass es einen bestimmten semantischen Einfluss hat, wird von Sudhoff (2003, S. 117ff.) hingewiesen, was auch hier in Abschnitt 3 gezeigt wird.

Anders als bei Sudhoff und Sternefeld ist bei Müller (1995), Frey (2011) und Zimmermann (i.Vorb.) bei fehlendem es-Korrelat nur der Komplementsatz Argument von $\mathrm{V}^{0}$ vgl. (19a) mit (15c). Die CP' besitzt nach Frey (2011) nominale Eigenschaften, so dass sie problemlos topikalisiert und extraponiert werden kann - vgl. (19b, c).

(19a) $\left[{ }_{\mathrm{CP}}\right.$ Max hat ... $\left[_{\mathrm{V}}, \mathrm{CP}\right.$ ' bedauert $]$

(19b) $\left[_{\mathrm{CP}} \mathrm{CP}^{\mathrm{i}} \mathrm{i}\right.$ hat Max $\ldots\left[{ }_{\mathrm{V}}, \mathrm{t}_{\mathrm{i}}\right.$ bedauert $]$

(19c) $\left[{ }_{\mathrm{CP}}\right.$ Max hat ... $\left[_{\mathrm{VP}}\left[{ }_{\mathrm{VP}}\left[{ }_{\mathrm{V}}, \mathrm{t}_{\mathrm{i}}\right.\right.\right.$ bedauert $\left.\left.\left.]\right] \mathrm{CP}_{\mathrm{i}}{ }_{\mathrm{i}}\right]\right]$

Damit haben Müller, Frey und Zimmermann Sudhoffs und Sternefelds Problem mit dem leeren es-Kopf umgangen. Ihnen bleibt aber die Frage, wann der Argumentsatz eines Verbs wie bedauern mit Korrelatkopf vorkommen kann und wann ohne.

\subsection{2 „Anaphorisches $e s^{\text {“ }}$}

Das anaphorische es-Korrelat betrachten Sudhoff (2003, i.Vorb.) und Frey (2011) als ein $\mathrm{V}^{0}$-Komplement im Mittelfeld, dessen Bezugssatz in der Rechtsversetzungsposition basisgeneriert ist - vgl. (20).

(20) Max behauptet es, dass Mia krank ist.

$$
\left[{ }_{\mathrm{CP}}\left[{ }_{\mathrm{CP}} \ldots\left[{ }_{\mathrm{V}}, \text { es ... }\right]\right] \mathrm{CP}^{\prime}\right]
$$

Das anaphorische es-Korrelat ist nicht auf eine bestimmte Unterklasse der Objekt-es-Verben beschränkt. Sowohl Verben wie behaupten und glauben erlauben es, als auch Verben wie bedauern und sich freuen. Letztere erlauben, wie in Abschnitt 2.2.1 gezeigt, auch das so genannte Platzhalter-es.

Bei dem Bezugssatz des anaphorischen es-Korrelats handelt es sich nach Sudhoff und Axel-Tober (2012) um einen quasiattributiven Gliedteilsatz, der an die Matrix-CP adjungiert ist, da er weder topikalisiert noch aus ihm extrahiert werden kann - vgl. (13b). Gegen die Auffassung, dass Konstruktionen wie (20) Rechtsversetzungskonstruktionen sind, spricht die Voranstellung des Verbalkomplexes:

(21) $\left[_{\mathrm{VP}} \text { Es behauptet haben, dass die Gruberova die Größte ist }\right]_{\mathrm{i}}$ kann nur MAx t. 
Kommen behaupten-Verben ohne es-Korrelat vor, ist bei Sudhoff und Frey der Komplementsatz wie bei den korrelatlosen bedauern-Verben ein $\mathrm{V}^{0}$-Komplement - vgl. (19a) und (22a). Aus dieser Position kann er sowohl topikalisiert als auch extraponiert werden:

(22a) Max hat, [dass Mia krank ist], behauptet.

(22b) [Dass Mia krank ist $]_{i}$, hat Max $t_{i}$ behauptet.

(22c) Max hat $t_{i}$ behauptet, [dass Mia krank ist $]_{i}$.

Und, er kann mit dem Verbalkomplex in die linke Peripherie bewegt werden - vgl. (23).

(23) $\left[_{V P} \text { Behauptet haben, dass er Mia gesehen hat }\right]_{i}$ kann nur FrANK $t_{i}$.

Fassen wir die bisherigen Argumente gegen die Homonymieansätze zusammen: Gegen die Rechtsversetzung des Bezugssatzes des es-Korrelats bei behaupten-Verben sprach erstens, dass der Bezugssatz mit dem Verbkomplex topikalisiert werden kann - vgl. (21). Es wird zweitens nicht gezeigt, welche Funktion das Platzhalter-es als „Determinierer“ hat und warum dieser mal overt oder nicht overt sein kann (Sudhoff 2003; Sternefeld 2006) beziehungsweise warum bei gleichem Matrixprädikat der Bezugssatz mit oder ohne Determinierer vorkommen kann (Müller 1995; Frey 2011; Zimmermann, i.Vorb.).

Ein weiteres Problem ergibt sich im Zusammenhang mit ergänzenden wenn-Sätzen, die wie ihr Korrelat F-markiert sein können - vgl. (24).

(24) Was gibt es Neues?

Stell dir vor, Max $\left[_{F}\right.$ bedauert $_{F} e_{F}$, [ ${ }_{F}$ wenn Italien spielt]].

Im Rahmen der Homonymiehypothese müsste das es-Korrelat, da es F-markiert ist, ein Platzhalter-es sein, also ein Kopf mit einem konditionalen Komplementsatz, was jedoch nicht plausibel ist. Außerdem könnte eine solche Annahme nicht erklären, warum das esKorrelat obligatorisch ist, wenn der wenn-Satz topikalisiert ist oder im Mittelfeld steht vgl. (25a, b) und (16b).

(25a) Wenn Mia kommt, bedauert*(es) Max.

(25b) Max hat *(es) immer, wenn Mia kam, bedauert.

Dass es sich bei dem es in Konstruktionen mit ergänzenden wenn-Sätzen eindeutig um eine Proform und bei seinem Bezugssatz um ein Adverbial handelt, wird bereits von Fabricius-Hansen (1980) gezeigt und auch von Sudhoff (2003), Kaiaty (2010) und Schwabe (i.Vorb.) angenommen.

$\mathrm{Zu}$ bedenken ist außerdem die Rolle von Korrelaten mit interrogativen Bezugssätzen wie (26). Hier müsste man einen Determinierer mit einem interrogativen Komplement postulieren.

(26) Was gibt es Neues?

Die Deutschen [ können es sich aussuchen $_{\mathrm{F}}$, F $_{\mathrm{F}}$ ob sie gegen Italien spielen]]

\subsection{3 "Nicht-thematisches $e s^{\text {“ }}$}

Sich auf Berman (2003), Cinque (1990) und Bennis (2004) beziehend sieht Frey (2011) außer dem Platzhalter-es und dem anaphorischen es ein drittes, von ihm nicht benanntes, es vor. Es entspricht dem Subjekt-es - vgl. (6). Frey weist diesem einen ,nicht-thematischen“ Argumentstatus zu. Er begründet diesen dritten Typ damit, dass das Subjekt-es im 
Mittelfeld obligatorisch ist, wenn die Verbalphrase samt Bezugssatz vorangestellt ist vgl. (27a, b), während das Objekt-es dort sehr markiert ist - vgl. (27c, d).

(27a) [Denkbar gewesen, dass Max kommt,] ist es für uns.

(27b) [Genervt/interessiert/verwundert, dass Max kommt], hat es sie.

(27c) ??[Bedauert, dass die Gruberova nicht die Größte ist,] hat es wohl Max.

(27d) ??[Geglaubt, dass die Gruberova nicht die Größte ist,] hat es wohl Max.

Eine weitere Besonderheit des Subjekt-es besteht nach Frey darin, dass es $w$-Extraktion nicht strikt verbietet - vgl. (28a, b) sowie Haider (2010, S. 208). Bei behaupten-Verben, die als Brückenverben eigentlich $w$-Extraktion erlauben, wird diese bekanntlich durch das es-Korrelat blockiert - vgl. (28c).

(28a) Wohin ${ }_{\mathrm{i}}$ hat (es) ihn denn gefreut/überrascht, dass sie $\mathrm{t}_{\mathrm{i}}$ gefahren ist?

(28b) Wohin $_{\mathrm{i}}$ ist (es) denkbar, dass Max $\mathrm{t}_{\mathrm{i}}$ gegangen ist?

(28c) *Wohin hat Frank es behauptet, dass Max gegangen ist?

Für Frey enthält der eingebettete Satz bei behaupten-Prädikaten ein Force-Merkmal, das die $w$-Extraktion lizensiert. Steht das es-Korrelat im Mittelfeld - es ist bei diesen Prädikaten, so Frey (2011), anaphorisch - hat der eingebettete Satz das Force-Merkmal nicht, was die $w$-Extraktion blockiert. Das Subjekt-es hat, so Frey (2011) und Haider (2010), diese blockierende Wirkung nicht. Hier ist zu bemerken, dass auch bestimmte Verben mit Objekt-es die $w$-Extraktion nicht verbieten:

(29a) Wohin zieht Max es vor, dass Lea reisen wird?

(29b) Wohin kann er es sich vorstellen, dass sie reisen wird?

Befunde wie die in (29) zeigen, dass die Möglichkeit der $w$-Extraktion kein Argument dafür darstellt, einen besonderen Status für das Subjekt-es anzunehmen.

Als ein weiteres Argument für den besonderen Status des Subjekt-es wird von Frey angeführt, dass es, vorausgesetzt das Prädikat lizensiert V2, V2-Einbettung nicht blockiert vgl. (30a, b). ${ }^{5}$ Behaupten-Verben haben ja bekanntlich diese Wirkung - vgl. (30c).

(30a) Laut Mia ist wäre es schon denkbar, Peter ist weggegangen.

(30b) Für Mia ist es denkbar, dass Peter sich wohl geirrt hat.

(30c) *Frank glaubt es, Mia ist krank.

Hier ist zu bedenken, dass Konstruktionen mit Subjekt-es und V2-Bezugssatz, ähnlich marginal sind wie Konstruktionen mit Objekt-es und V2-Bezugssatz - vgl. die Beispiele unter (2). Die indikativische Version von (30a) ?? Laut Mia ist es denkbar, Peter ist weggegangen ist fragwürdig.

Laut Frey besteht der besondere Status des Subjekt-es also darin, dass es anders als das Objekt-es bei der Voranstellung des Verbalkomplexes obligatorisch und bei der V2- und Modalpartikeleinbettung möglich ist. Mit dem von bedauern-Verben lizensierten Platzhalter-es hat das Subjekt-es gemeinsam, dass es nicht referiert und dass es F-markiert sein kann. Oben wurde bereits zu bedenken gegeben, dass es nicht erwiesen ist, dass das Subjekt-es V2 toleriert, wie auch nicht, dass es wie das es-Korrelat von bedauern-Verben nicht referiert. Wie der folgende Abschnitt zeigen wird, beruht der besondere Status des

(30a) und (30b) entsprechen Freys (2011) Beispielen (6b) und (7b). Freys Beispiel (6b) heißt wörtlich Laut Mia ist es denkbar, dass wir Peter eben doch einladen. 
Subjekt-es bei der Voranstellung des Verbalkomplexes darauf, dass es sich wie auch ein alternatives pro in SpecI befinden muss, letzteres jedoch in einer bestimmten syntaktischen Relation zu seinem Bezugssatz stehen muss. Zieht man die hier vorgebrachten Einwände und alternativen Lösungen im folgenden Abschnitt in Betracht, erscheint der Schluss, dass es sich bei dem Subjekt-es um einen speziellen es-Typ handelt, als nicht zwingend.

Dass es sich bei dem Subjekt-es, anders als von Frey angenommen, um eine referenzielle Proform handelt, macht zudem wieder die ergänzende wenn-Form deutlich. Das Subjektes ist obligatorisch, wenn der Konditionalsatz vorangestellt ist.

(31) Wenn Mia KRANK ist, ist es für Max bedauerlich.

Im Folgenden werde ich für ein uniformes $e s$-Korrelat plädieren, eine Proform, die auf eine kontextgegebene Aussage referiert.

\section{$2.3 \quad$ Uniformes es}

Ähnlich wie Haider (2010, S. 203ff.) gehe ich davon aus, dass ein Komplementsatz im Mittel- oder Nachfeld basisgeneriert ist. ${ }^{6}$ Im Mittelfeld befindet er sich in den jeweilig kanonischen Argumentpositionen. Als Subjektsatz ist er in SpecvP und als direkter Objektsatz ist er ein Komplement von $\mathrm{V}^{0}-$ vgl. (32a-c). In diesen Positionen ist er direkt von $\mathrm{V}^{0}$ lizensiert und kann von dort, ohne das ECP zu verletzen, ins Vorfeld gehen. ${ }^{7}$

(32a) Mia hat doch, dass Peter kommt, geglaubt/abgelehnt, oder?

$\left[{ }_{\mathrm{CP}} \cdots\left\{_{\mathrm{V}}, \mathrm{CP}^{\prime}\left[{ }_{\mathrm{V} 0}\right.\right.\right.$ geglaubt $\left.\left.]\right\}\right]$

(32b) Vielleicht hat ihn, dass Peter kommt, interessiert.

$\left[{ }_{\mathrm{CP}} \ldots \mathrm{ihn}_{\mathrm{i}} \ldots\left\{_{\mathrm{vP}} \mathrm{CP}, \ldots\left[{ }_{\mathrm{V}}, \mathrm{t}_{\mathrm{i}}\left[{ }_{\mathrm{V} 0}\right.\right.\right.\right.$ interessiert $\left.\left.\left.]\right]\right\}\right]$

(32c) Mia hat, dass Max kommt, gut gefunden, dass Leo kommt hingegen nicht.

$\left[{ }_{\mathrm{CP}} \cdots\left\{_{\mathrm{V}}, \mathrm{CP}^{\prime}\left[_{\mathrm{V} 0}[\mathrm{AP}\right.\right.\right.$ gut $]$ gefunden $\left.\left.]\right\}\right]$

Ist CP' in der Extraposition, ist er an VP basisadjungiert - vgl. (33a-c). Die Komplementposition ist entweder mit einem es-Korrelat oder pro besetzt, die dort theta-markiert und damit direkt lizensiert sind - vgl. Schwabe (i.Vorb.).

(33a) Mia hat (es) geglaubt/abgelehn/bedauert, dass Peter kommt.

${ }_{\mathrm{CP}} \cdots\left\{_{\mathrm{VP}}\left[{ }_{\mathrm{VP}}\left[{ }_{\mathrm{V}}\right.\right.\right.$, pro/es geglaubt $\left.\left.\left.]\right] \mathrm{CP}^{\prime}\right\}\right]$

(33b) Vielleicht hat (es) ihn interessiert, dass Peter kommt. $\left[{ }_{\mathrm{CP}}\right.$ vielleicht $\ldots\left[_{\mathrm{IP}}\right.$ pro $_{\mathrm{i}} e s_{\mathrm{i}}\left[_{\mathrm{vP}} \mathrm{t}_{\mathrm{i}} \ldots\left\{_{\mathrm{VP}}\left[{ }_{\mathrm{VP}}[\mathrm{V}\right.\right.\right.$, ihn interessiert $\left.\left.\left.\left.\left.]\right] \mathrm{CP}\right\}\right]\right]\right]$

(33c) Mia hat (es) gut gefunden, dass Peter kommt.

$\left[{ }_{\mathrm{CP}} \mathrm{Mia} \ldots\left\{_{\mathrm{VP}}\left[{ }_{\mathrm{VP}} \ldots\left[{ }_{\mathrm{V}}\right.\right.\right.\right.$, pro/es $\left[{ }_{\mathrm{V} 0}\left[{ }_{\mathrm{AP}}\right.\right.$ gut $]$ gefunden $\left.\left.\left.\left.]\right]\right] \mathrm{CP}\right\}\right]$

Der Komplementsatz in der Extraposition ist nur indirekt lizensiert und kann deshalb von dort auch nicht topikalisiert werden - vgl. Haider (2010, S. 227). Seine Topikalisierung würde zu einer ECP-Verletzung führen.

Das es-Korrelat ist eine DP, da es durch sein flektierbares, starkes Pendant, das das, ersetzt werden kann - vgl. Mia ist sich dessen bewusst, dass Leo krank ist. Da es sich bei ihm um eine schwache Proform handelt, kann es nicht im Vorfeld stehen - vgl. (34a-c).

6 Komplementsätze im Mittelfeld sind in der Regel stark markiert, sie kommen dort aber unter bestimmten Bedingungen vor, wie (32) zeigt.

7 Vgl. „Empty Category Principle“ bei Chomsky (1986, S. 250), wonach eine leere Kategoie regiert sein muss. 
(34a) *Es bedauert/glaubt Frank, dass Mia krank ist.

(34b) *Es findet Frank richtig, dass Mia krank ist.

(34c) *Es hat er kommen sehen, dass Mia krank ist.

Bei (35a) ist, anders als von Meinunger (2007) für ähnliche Beispiele behauptet, nicht ein Objekt-es topikalisiert, sondern die satzinitiale Position ist von einem positionalen es besetzt. (35b) zeigt, dass außer dem positionalen es zusätzlich noch ein es-Korrelat vorkommen kann. Die Objektstelle in (35a) nimmt ein pro ein.

(35a) Es wisse doch jeder, dass die Karriere davon abhänge, dass man „dem Alten nicht widerspricht“". (IDS brz 2010)

$\left[{ }_{C P}\right.$ es wisse $_{i} \ldots\left[_{V P}\left[{ }_{V P}\right.\right.$ pro t $\left.\left.\left.{ }_{i}\right] C^{\prime}{ }^{\prime}\right]\right]$

(35b) Ich will nicht weiter nachforschen, wo diese plötzliche Liebe zur Südschweiz ihre Wurzeln hat; es weiß es ohnehin jeder, (wo ...) (DWDS Zeit 1959)

Wie das Objekt-es kann auch das Subjekt-es nicht im Vorfeld erscheinen. Befindet sich dort ein es-Lexem, handelt es sich wieder um ein positionales es. Das wird in $(36 \mathrm{a}, \mathrm{b})$ deutlich, wo neben dem positionalen es noch ein Subjekt-es figuriert. Demnach handelt es sich bei dem es in (36c) um ein positionales es und die Komplementposition ist durch ein pro besetzt.

(36a) Sie [Frauen] sehen deshalb gut im Nahbereich, aber es ist es schwerer für sie, Wild zu entdecken. (DWDS BZ 2005)

$\left[{ }_{\mathrm{CP}}\right.$ es ist $\mathrm{es}_{\mathrm{j}} \ldots\left[_{\mathrm{vP}} \mathrm{t}_{\mathrm{j}} \ldots\left\{_{\mathrm{VP}}\left[{ }_{\mathrm{VP}}\left[\mathrm{V}\right.\right.\right.\right.$, schwer $\left.\left.\left.\left.\left._{\mathrm{i}}\right]\right] \mathrm{CP}^{\prime}\right\}\right]\right]$

(36b) Ein Helfer reicht ihm den Rucksack und, siehe da, es ist es gar kein Rucksack, sondern ein Wunder moderner Technik! (DWDS BZ 2003)

$\left[\mathrm{CP}\right.$ es ist $\left._{\mathrm{i}} \mathbf{e s}_{\mathrm{j}} \ldots\left[_{\mathrm{vP}} \mathrm{t}_{\mathrm{j}} \ldots\left\{_{\mathrm{VP}}\left[\mathrm{VP}\left[\mathrm{V}, \mathrm{e}_{\mathrm{i}}\right]\right] \mathrm{CP}\right\}\right]\right]$

(36c) Es war für ihn ganz schwer, dass er das einsehen musste. (DWDS Zeit 2001)

$\left[{ }_{\mathrm{CP}}\right.$ es war $_{\mathrm{i}}$ pro $_{\mathrm{j}} \ldots\left[_{\mathrm{vP}} \mathrm{t}_{\mathrm{j}} \ldots\left\{_{\mathrm{VP}}\left[{ }_{\mathrm{VP}}\left[{ }_{\mathrm{V}}\right.\right.\right.\right.$, ganz schwer $\left.\left.\left.\left.\left.\left.\mathrm{e}_{\mathrm{i}}\right]\right] \mathrm{CP}\right\}\right\}\right]\right]$

Daraus, dass das Subjekt- und das Objekt-es nicht akzentfähig sind, folgt auch, dass sie, anders als ihr akzentfähiges Pendant das, nicht zusammen mit ihrem Bezugssatz eine Konstituente bilden und so im Mittelfeld oder Vorfeld erscheinen können - vgl. (37a, b).

(37a) *Frank hat \{es [ dass Mia krank ist]\} bedauert.

(37b) Frank hat \{das [dass Mia krank ist]\} bedauert.

Die hier vorgestellte Analyse kann, ohne drei Korrelatkategorien zu postulieren, erklären, warum der Bezugssatz nicht topikalisiert sein kann, wenn sich im Mittelfeld ein es-Korrelat befindet - vgl. $(38 \mathrm{a}, \mathrm{b})$ und $(16 \mathrm{a}, \mathrm{b})$.

(38a) [Dass Mia krank ist $]_{i}$, bedauert $t_{i}$ Frank.

(38b) *[Dass Mia krank ist], bedauert es Frank.

Ist der Bezugssatz wie in (38a) in der kanonischen Komplementposition generiert, ist in dieser Konstellation für ein Korrelat kein Platz - vgl. (38b). Ist der Bezugssatz wie in (33a-c) in der Extraposition generiert, ist er durch sein es-Korrelat oder pro, die direkt von $\mathrm{V}^{0}$ lizensiert sind, indirekt lizensiert. Da Topikalisierung nur aus einer direkt lizensierten Position möglich ist, kann CP' nicht aus dem Nachfeld heraus bewegt werden.

Der uniforme Ansatz kann ebenfalls erklären, warum das Subjekt-es anders als das Objekt-es obligatorisch ist, wenn der Verbalkomplex vorangestellt wird - vgl. (27a-d) und (39a', b'). 
(39a) Durchgesickert, dass er sich geirrt hat, ist es gestern.

$\left[{ }_{\mathrm{CP}}\left\{{ }_{\mathrm{VP}}[\mathrm{VP}\right.\right.$ durchgesickert $\left.] \mathrm{CP}^{\prime}\right\} \mathrm{j}$ ist $\left.\ldots\left[_{\mathrm{IP}} \mathbf{e s}_{\mathrm{i}}\left[{ }_{\mathrm{I}} \ldots\left[_{\mathrm{VP}} \mathrm{t}_{\mathrm{i}} \ldots \mathrm{t}_{\mathrm{j}}\right]\right]\right]\right]$

(39a')*Durchgesickert, dass er sich geirrt hat, ist pro gestern.

$\left[{ }_{\mathrm{CP}}\left\{\left\{_{\mathrm{VP}}\left[{ }_{\mathrm{VP}}\right.\right.\right.\right.$ durchgesickert $\left.] \mathrm{CP}\right\} \mathrm{j}$ ist $\ldots$ [IP pro $\left.\left.\left.{ }_{\mathrm{i}}, \ldots\left[{ }_{\mathrm{vP}} \mathrm{t}_{\mathrm{i}} \ldots \mathrm{t}_{\mathrm{j}}\right]\right]\right]\right]$

(39b) $\left[_{\mathrm{CP}}\left\{\left\{_{\mathrm{VP}}\left[{ }_{\mathrm{VP}}\left[{ }_{\mathrm{V}}, \mathrm{t}_{\mathrm{i}}\right.\right.\right.\right.\right.$ bedauert $\left.\left.]\right] \mathrm{CP}\right\}$ j hat $\mathbf{e s}_{\mathrm{i}} \ldots\left[_{\mathrm{VP}}\right.$ Frank $\left.\left.\ldots \mathrm{t}_{\mathrm{j}}\right]\right]$

$\left(39 b^{\prime}\right)\left[_{C P}\left\{_{V P}\left[\left[_{V P}\left[{ }_{V}, \text { pro bedauert }\right]\right] C^{\prime}\right\}_{j}\right.\right.$ hat $\ldots\left[_{I P}\right.$ Frank $\left.\left._{\mathrm{i}}\left[_{\mathrm{VP}} \mathrm{t}_{\mathrm{i}} \ldots \mathrm{t}_{\mathrm{j}}\right]\right]\right]$

Der unmögliche Kongruenzabgleich zwischen dem Subjekt-pro in SpecI und finitem Verb in $I^{0}$ allein kann es nicht sein, denn (33b) zeigt, dass bei der Extraposition SpecI durch ein pro besetzt sein kann. Ein ähnliches Problem zeigt sich nach Schwabe (i.Vorb.) bei der Voranstellung von ergänzenden wenn-Sätzen. Pro ist dort nicht lizensiert, anders als bei der Extraposition:

(40a) [Wenn Mia krank ist $]_{k}$ bedauert ${ }_{j} \mathbf{e s}_{i}\left[{ }_{I P} t_{k}\left[{ }_{I P} \operatorname{Max} \ldots\left[\left[_{V P}\left[{ }_{V}, t_{i} t_{j}\right]\right]\right]\right]\right.$

(40b) *[Wenn Mia krank ist $]_{\mathrm{k}}$ bedauert ${ }_{\mathrm{j}} \mathrm{L}_{\mathrm{IP}} \mathrm{t}_{\mathrm{k}}\left[_{\mathrm{IP}} \operatorname{Max} \ldots\left[_{\mathrm{VP}}\left[\mathrm{V}_{\mathrm{V}}\right.\right.\right.$, pro $\left.\left.\left.\left.\mathrm{t}_{\mathrm{j}}\right]\right]\right]\right]$

(40c) Max bedauert ${ }_{\mathrm{j}} \ldots\left\{_{\mathrm{VP}}\left[_{\mathrm{VP}}\left[{ }_{\mathrm{V}}\right.\right.\right.$, pro $\left.\left.\mathrm{t}_{\mathrm{j}}\right]\right]$ wenn Mia krank ist $\}$

Sowohl pro als auch es sind syntaktisch lizensiert, wenn sie von $\mathrm{V}^{0}$ theta-markiert sind. Für pro gilt zusätzlich, dass es seinen Bezugssatz $\mathrm{m}_{\mathrm{e}}$-kommandieren muss. $\mathrm{M}_{\mathrm{e}}$-Kommando ist nach Pesetsky (1991, S. 67) wie folgt definiert:

(41) $m_{e}$-Kommando

$\alpha \mathrm{m}_{\mathrm{e}}$-kommandiert $\beta$, gwd. $\alpha \beta$ nicht dominiert und keine maximale Projektion $\gamma$, die $\alpha$ dominiert, $\beta$ ausschließt.

(41a) $\alpha$ ist von $\beta$ dominiert, gdw. es von jedem Segment von $\beta$ dominiert ist.

(41b) $\alpha$ schließt $\beta$ aus, wenn kein Segment von $\alpha \beta$ dominiert.

In (39a') und (40b) ist diese Bedingung nicht erfüllt, denn der dass- beziehungsweise der wenn-Satz sind aus der VP ausgeschlossen und können somit von pro nicht $\mathrm{m}_{\mathrm{e}}$-kommandiert werden. In (39b') hingegen befinden sich sowohl pro als auch der Bezugssatz in der VP, so dass das $\mathrm{m}_{\mathrm{e}}$-Kommando erfüllt ist. Zu erklären bleiben letztlich die Fokus-, V2und $w$-Extraktionsdaten in (12) und (13), (3) und (28-30). Wir kommen in Abschnitt 3 darauf zurück.

\section{$2.4 \quad$ Uniforme ProPP}

Eine ProPP im Mittelfeld ist von $\mathrm{V}^{0}$ direkt lizensiert - vgl. (42a). Der Bezugssatz der ProPP ist wie der des Objekt- und Subjekt-es im Nachfeld als VP-Adjunkt basisgeneriert und als dieses nicht direkt lizensiert. Auch hier schließt das ECP aus, dass der Bezugssatz in das Vorfeld bewegt wird - vgl. (42b).

(42a) Frank hat sich daRüber/DRüber gefreut, dass Mia gesunD ist.

$$
\left[{ }_{\mathrm{CP}} \cdots\left\{_{\mathrm{VP}}\left[{ }_{\mathrm{VP}} \cdots\left[_{\mathrm{V}},\left[_{\mathrm{PP}} \text { darüber }\right]\left[_{\mathrm{V} O} \text { gefreut }\right]\right]\right] \mathrm{CP}\right\}\right]
$$

(42b) *[Dass Mia krank ist $]_{\mathrm{i}}$, hat sich Frank darüber gefreut $\mathrm{t}_{\mathrm{i}}$.

Der Bezugssatz kann, da der pronominale Teil der ProPP akzentfähig ist, an die ProPP adjungiert sein. Diese komplexe PP kann sowohl im Mittel- als auch Vorfeld erscheinen - vgl. (43a, b). Der Bezugssatz kann nicht aus der PP heraus topikalisiert werden, da dies zu einer Inselverletzung führen würde - vgl. (43c) und (16a). 
(43a) Frank hat sich $\left\{_{\mathrm{PP}}\right.$ DArüber [dass Mia gesund ist]\}, gefreut.

(43b) $\left\{_{\mathrm{PP}}\right.$ DArüber [dass Mia gesund ist]\}, hat sich Frank gefreut.

$(43 \mathrm{c}) *[\text { dass Mia gesund ist }]_{\mathrm{i}}$ hat sich Frank $\left\{_{\mathrm{PP}}\right.$ darüber $\left.\mathrm{t}_{\mathrm{i}}\right\}$, gefreut.

In Abschnitt 2.1.3 haben wir gesehen, dass bei bestimmten Matrixprädikaten der Bezugssatz im Nachfeld figurieren kann, ohne dass sich im Mittelfeld eine overte ProPP befindet. Analog zum pro für ein es-Korrelat kann auch für eine fehlende ProPP ein pro stehen. vgl. (44a) und (9a'-c'). Dieses pro ist wieder durch $\mathrm{V}^{0}$ lizensiert und dadurch, dass es seinen Bezugssatz $\mathrm{m}_{\mathrm{e}}$-kommandiert - vgl. (41) und (44a). Da pro nicht akzentuierbar ist, kann es nicht zusammen mit seinem Bezugssatz eine Konstituente bilden - vgl. (44b, c).

(44a) Frank hat sich $\left[{ }_{\mathrm{VP}}[\mathrm{VP}\right.$... pro gefreut $]$ dass Mia kommt $]$

(44b) *Frank hat sich \{ pro [dass Mia kommt]\} gefreut.

$(44 c) *\{$ p pro [dass Mia kommt $]\}_{\mathrm{i}}$ hat sich $\mathrm{t}_{\mathrm{i}}$ Frank gefreut.

Müller (1995, S. 230ff.), Sternefeld (2006, S. 359) und Axel-Tober (2012, S. 55f.) behandeln ProPP-lose Konstruktionen analog zu ProPP-haltigen. Die ProPP ist bei ihnen ein Kopf, der zusammen mit dem Bezugssatz eine Konstituente bildet. Diese komplexe PP kann sowohl im Mittel- als auch im Vorfeld figurieren - vgl. (43a, b). Ist der Bezugssatz extraponiert, kann die ProPP overt, aber auch getilgt sein - vgl. (45).

(45) Frank hat sich darüber/ $\varnothing$ gefreut, dass Mia gesund ist.

$\left[_{\mathrm{CP}} \cdots\left\{_{\mathrm{VP}}\left[{ }_{\mathrm{VP}} \cdots\left[_{\mathrm{V}},\left\{_{\mathrm{PP}}\right.\right.\right.\right.\right.$ darüber/ $\left.\varnothing \mathrm{t}_{\mathrm{i}}\right\}$ gefreut $\left.\left.\left.]\right] \mathrm{CP}_{\mathrm{i}}\right\}\right]$

Die Ansätze der oben genannten Autoren sind dem hier propagierten sehr ähnlich. Sie unterscheiden sich jedoch von dem hiesigen darin, dass letzterer eine nicht realisierte ProPP als pro ansieht, das allein steht, das heißt, das kein Kopf einer komplexen PP ist - vgl. (44a) mit (45). Dass eine komplexe PP mit leerem Kopf nicht topikalisiert werden kann, erklärt Axel-Tober damit, dass Konstituenten mit leeren Köpfen generell nicht topikalisierbar sind.

Gegen die Annahme eines getilgten P-Kopfes spricht, dass nicht klar ist, wodurch die ProPP-Tilgung bedingt ist. Fehlende Akzentfähigkeit wie bei dem es-Korrelat kann es nicht sein, denn eine ProPP ist akzentfähig. Sie muss sogar akzentuiert sein, wenn sie zusammen mit ihrem Bezugssatz eine Konstituente bildet. ${ }^{8}$ Hinzu kommt, dass nicht erläutert wird, warum die einen Prädikate ProPP-Tilgung erlauben und die anderen nicht - vgl. darunter leiden vs. sich (darüber) freuen. Im Hinblick auf die Annahme eines leeren Kopfes stellt sich auch die Frage, warum er im Mittelfeld nicht möglich ist.

Dass der pronominale Teil einer ProPP immer dann akzentuiert ist, wenn, wie in (43a, b), die ProPP zusammen mit ihrem Bezugssatz eine Konstituente bildet, ist schon von Breindl (1989) beobachtet worden. Sie weist darauf hin, dass wenn ProPP und Bezugssatz dissoziiert sind, der pronominale Teil der ProPP keinen Akzent zu tragen braucht - vgl. (42a). Bei einigen Prädikaten kann die ProPP sogar reduziert sein - vgl. sich drüber freuen, sich drauf stützen und drauf warten. Nach Breindl (1989, S. 189f.) gibt es Prädikate, bei denen der pronominale Teil der ProPP den Hauptakzent tragen muss und damit nicht reduzierbar ist. $\mathrm{Zu}$ diesen zählen relationale Prädikate wie darin bestehen, darin/daran liegen, sich darin/daran zeigen, sich darin ausdrücken, sich darin erweisen, darauf beruhen, sich darin bekunden, damit zusammenhängen und sich daraus ergeben sowie Prädikate, bei denen das Komplement einem Modifikator zu entsprechen scheint - vgl. daran erkennen und

An dieser Stelle sei an das Sudhoffs (2003, i.Vorb.) Problem bei der Analyse des Platzhalter-es erinnert. Bei ihm kann nur eine DP mit leerem Kopf topikalisiert werden - vgl. (17b). 
daran merken. Bei all diesen Prädikaten ist die ProPP obligatorisch. Die Behauptung, dass bei diesen Verben der Hauptakzent auf dem pronominalen Element der ProPP liegen muss, scheint durch die folgenden Beispiele infrage gestellt.

(46a) „Die Gunst ein Potsdamer zu sein, besteht darin, jederzeit durch diesen PARK wandeln zu können“, schreibt Fritz-Jochen Kopka über Sanssouci. (DWDS BZ 2005)

(46b) Die Schwierigkeit dieses Falles lag daRin, daß die Tat - Totschlag - nach 20 Jahren in der Bundesrepublik verJÄHRT war, nicht aber nach DDR-Recht. (DWDS BZ 1997)

In den Beispielen unter (46) befindet sich der Hauptakzent auf dem Fokusexponenten des Bezugssatzes. Der pronominale Teil der ProPP trägt nicht den Hauptakzent, ist jedoch, wie Breindl zu Recht feststellt, nicht reduzierbar. Was zu erklären ist, ist also, warum es Verben gibt, bei denen der pronominale Teil nicht reduziert werden kann.

Aufgrund des Kontrasts, dass der pronominale Teil einer ProPP bei bestimmten Verben immer den Hauptakzent trägt, bei anderen nicht, unterscheidet Breindl (157ff.) zwischen Bezugselementen und Platzhaltern. Ein Bezugselement ist bei ihr ein Korrelat, bei dem der pronominale Teil prominent akzentuiert ist. Es ist anaphorisch, referiert also, und sein Bezugssatz ist ein quasiattributiver Gliedteilsatz. Ein Platzhalter ist ein Korrelat, dessen pronominaler Teil nicht prominent akzentuiert ist. Er referiert nicht und sein Bezugssatz ist ein Gliedsatz - vgl. auch Reis (1997) und Axel-Tober (2012). Da, so Breindl (S. 187), bei Verben wie darin bestehen und damit zusammenhängen der pronominale Teil der ProPP immer den Hauptakzent trägt, kommen diese Verben immer nur mit einem Bezugselement vor. Nun zeigt aber (46), dass bei diesen Verben der pronominale Teil bestenfalls nicht reduzierbar ist. $\mathrm{Ob}$ diese Beobachtung dafür ausreicht, zwischen einer Platzhalteroder einer Bezugselement-ProPP zu unterscheiden, kann bezweifelt werden - vgl. auch Zifonun/Hoffmann/Strecker (1997, S. 1488).

Wir gehen davon aus, dass der pronominale Teil einer ProPP wie das es-Korrelat eine Proform ist, die sich auf eine Aussage bezieht. Die ProPP ist bei bestimmten Prädikaten optional. Der nächste Abschnitt zeigt, wie die Verwendung eines optionalen Korrelats die Bedeutung eines Matrixprädikats modifizieren kann.

\section{Semantischer Effekte von Korrelaten}

In Abschnitt 2 wurde gezeigt, dass die Anwesenheit eines es-Korrelats V2-Einbettung und $w$-Extraktion verhindern kann - vgl. (13a, b) und (28c). Es wurde auch darauf hingewiesen, dass das es-Korrelat bei Prädikaten wie bedauern F-markiert sein kann, bei Prädikaten wie behaupten hingegen nicht - vgl. (12a, b). Außer diesen lassen sich noch weitere korrelatbedingte Effekte feststellen. So können nur die korrelatlosen Sätze (47a, b) durch einen Konzessivsatz fortgesetzt werden, ohne dass das ganze Gefüge, wie in (47a', b') zu sehen, inkonsistent wird.

(47a) Leo bedauert, dass Jan verloren hat, obwohl Jan eigentlich gewonnen hat.

(47a') *Leo bedauert es, dass Jan verloren hat, obwohl Jan eigentlich gewonnen hat.

(47b) Leo ärgert sich, dass Jan verloren hat, obwohl Jan eigentlich gewonnen hat.

$(47 b$ ')*Leo ärgert sich darüber, dass Jan verloren hat.

Das korrelatlose Beispiel (48a) kann mit Frank weiß, dass Max gewonnen hat oder Frank weiß, dass Max nicht gewonnen hat paraphrasiert werden - vgl. Hintikka (1976) und Schwabe/Fittler (2009, 2012). Bei dem ProPP-haltigen Beispiel (48b) hingegen ist diese Paraphrase nicht möglich. 
(48a) Frank hört, ob Max gewonnen hat.

(48b) Frank hört davon, ob Max gewonnen hat.

Die Anwesenheit eines es-Korrelats beziehungsweise einer ProPP wie in (49b, c) schließt die für (49a) mögliche exhaustive Interpretation Für alle $x$, Frank weiß ob x gewonnen hat aus - vgl. Schwabe/Fittler (2012).

(49a) Frank weiß, wer gewonnen hat.

(49b) Frank weiß es, wer gewonnen hat.

(49c) Frank weiß davon, wer gewonnen hat.

Im Folgenden versuche ich zu zeigen, wie sich die genannten Effekte erklären lassen. ${ }^{9}$ Dazu untersuchen wir, welche semantische Funktion Korrelate haben und wie sie mit der Verbbedeutung und bestimmten informationsstrukturellen Aspekten interagieren.

\subsection{Korrelate und eingebettete Deklarativsätze}

Ein eingebetteter Deklarativ, sei es ein dass- oder V2-Satz, referiert wie das Radikal eines Konditionals auf eine Aussage $\sigma$. Diese fasse ich mit Asher (1993) als ein abstraktes Objekt auf. Ist ein dass-Satz von einem inhärent veridikalen Verb wie wissen und beweisen eingebettet, ist die von ihm denotierte Aussage wahr, wenn der Gesamtausdruck wahr ist. ${ }^{10}$ Verben wie bedauern und sich freuen hingegen können auf falsche nicht-kontradiktorische und wahre nicht-tautologische Aussagen zutreffen, das heißt, nur auf Ereignisrepräsentationen. Verben wie behaupten oder annehmen, können auch für kontradiktorische Aussagenargumente gelten - vgl. Schwabe/Fittler (2012).

Es-Korrelate, der pronominale Teil von ProPPn wie auch pro referieren als Proformen auf eine Aussage. Ist die Aussage diskursgegeben, sind diese Proformen anaphorisch. Die Aussage kann direkt oder indirekt durch den Bezugssatz bezeichnet werden. Ein Bezugssatz bezeichnet die Aussage direkt, wenn es sich bei ihm um einen Deklarativ- oder Konditionalsatz handelt - vgl. (1a) und (1d). Der Bezugssatz und die Proform sind koindiziert, was gewährleistet, dass sich beide auf dieselbe Aussage beziehen - vgl. Schwabe (i.Vorb.). Handelt es sich bei dem Bezugssatz um einen Interrogativ, wird der Referent der Proform durch den Bezugssatz indirekt bezeichnet.

Es-Proformen beziehungsweise optionale ProPPs können die Bedeutung bestimmter Matrixverben modifizieren - vgl. Schwabe/Fittler (2012). So wird, wie auch von Sudhoff (2003, S. 117ff.) bemerkt, bedauern dass durch den Gebrauch des Korrelats faktiv. Das heißt, dass unabhängig davon, ob der Matrixsatz gilt oder nicht, der eingebettete Satz $\sigma$ wahr ist - vgl. Ėgré (2008). Zu den Verben, die durch eine es-Proform faktiv werden, zählen wissen dass, hören dass und bedauern dass, nicht aber beweisen dass und erreichen dass. Sich freuen dass wird ebenfalls durch den Gebrauch der ProPP faktiv.

Verben wie wissen, erfahren, hören und glauben lizensieren, wie wir in Abschnitt $2.1 \mathrm{im}$ Zusammenhang mit den Beispielen (11a,b) und in Abschnitt 3.1 gesehen haben, neben dem es-Korrelat auch eine ProPP. Bei davon wissen dass, davon erfahren dass und davon hören dass referiert die ProPP auf die Aussage $\sigma$, die durch den eingebetteten Deklarativ bezeichnet wird. Die Aussage $\sigma$ folgt aus dem, was das Matrixsubjekt weiß, erfährt oder

\footnotetext{
$9 \quad$ Auf die Diskussion des Einflusses von es-Korrelaten auf die $w h$-Extraktion wird hier verzichtet, da die Datenlage noch nicht ausreichend geklärt ist.

10 Ein Prädikat ist veridikal, wenn aus $\alpha$ Prädikat dass $\sigma$ folgt, dass $\sigma$ gilt.
} 
hört, was aber nicht heißen muss, dass sie vom Subjekt selbst gewusst, erfahren oder gehört werden muss. ${ }^{11}$ Das Subjekt weiß, erfährt oder hört aber von $\sigma$. Wissen und erfahren, die inhärent veridikal sind, werden durch den Gebrauch der ProPP faktiv. Hören hingegen, das nicht inhärent veridikal ist, wird es nicht. Das heißt, wenn Max (nicht) davon hört, dass Mia krank ist, muss sie nicht unbedingt krank sein.

\subsection{F-markierte es-Korrelate}

Dass die es-Proform in einem Matrixsatz mit einem bedauern-Prädikat F-markiert sein kann und bei einem behaupten-Prädikat nicht, gab Anlass zu der in Abschnitt 2.2 diskutierten und kritisierten Homonymiehypothese, nach der zwischen Platzhalter-es und anaphorischem es-Korrelat unterschieden wird - vgl. (12a, b) bzw. (50a, b).

(50) Was gibt es Neues?

(50a) ${ }_{\mathrm{F}}$ Max bedauert ${ }_{\mathrm{F}} \mathrm{es}_{\mathrm{F}}\left[\mathrm{F}\right.$ dass Mia KRANK $_{\mathrm{F}}$ ist $]$

(50b) $*{ }_{\mathrm{F}}$ Max behauptet ${ }_{\mathrm{F}} \mathrm{es}_{\mathrm{F}}\left[\right.$ dass $_{\mathrm{F}}$ Mia KRANK $_{\mathrm{F}}$ ist $\left.]\right]$

Was befähigt nun Verben wie bedauern dass, eine nicht-anaphorische es-Proform zuzulassen? Ist es die Faktivität, die bei bedauern durch das Korrelat induziert wird? Der Vergleich von bedauern und wissen zeigt, dass das es-Korrelat bei diesen Verben zwar Faktivität induziert, dass diese aber keine hinreichende Bedingung für die F-Markierung des es-Korrelats darstellt - vgl. (50a) und (51).

(51) Was gibt es Neues?

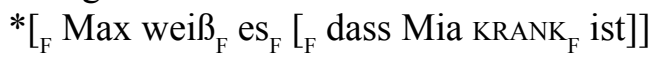

Faktivität ist auch keine notwendige Bedingung, denn veridikale, nicht-faktive Verben wie initiieren und erreichen können auch mit einer F-markierten es-Proform vorkommen:

(52) Was gibt es Neues über Italien?

Monti hat es hinbekommen/erreichen/initiiert, dass sich die Finanzlage stabilisiert.

Der Unterschied zwischen bedauern- und behaupten-Verben lässt sich mit Hilfe von Schwarzschilds (1999) Fokustheorie und Stalnakers (2002) Common Ground-Konzept beantworten. ${ }^{12}$ Nach Schwarzschilds (1999) Prinzip AVOIDF ist eine Phrase nur dann F-markiert, wenn es nötig ist und GIVENNESS nicht verletzt ist. GIVENNESS besagt, dass eine Äußerung U dann als gegeben (,given“) zählt, wenn es eine saliente anaphorische Äußerung A gibt und U und A koreferent sind. In (53a-c) ist AVOIDF erfüllt.

(53) Mia ist krank?

(53a) $\operatorname{Max}_{\mathrm{F}}\left[_{\mathrm{F}}\right.$ BEDAUERT $_{\mathrm{F}}$ es], dass Mia krank ist

(53b) $\operatorname{Max}_{\mathrm{F}}$ [ $_{\mathrm{F}}$ BEHAUPTET $_{\mathrm{F}}$ es], dass Mia krank ist

(53c) $\operatorname{Max}_{\mathrm{F}}\left[_{\mathrm{F}}\right.$ hat es GEHÖRT $\mathrm{F}$, dass Mia krank ist

Dass potenziell faktive Prädikate wie bedauern und veridikale Prädikate wie erreichen F-markierte es-Korrelate lizensieren, steht im Zusammenhang mit der Frage, wann eine Aussage zum Update eines Common Grounds werden kann. Unter dem Common Ground ist nach Stalnaker (2002) das von Diskursteilehmern geteilte Wissen zu verstehen. Erweitert eine Aussage einen Common Ground, handelt es sich bei ihr um ein Update dieses

${ }_{11}$ Schwabe/Fittler (2012) bezeichnen diese Erweiterung als Deduktiven Abschluss.

12 Hier werden nur informationsstrukturelle Eigenschaften von es-Korrelaten diskutiert, die von ProPPn werden vorerst nicht berücksichtigt. 
Common Grounds. Wir sehen eine Aussage als ein potenzielles Update an, wenn bei ihrer sprachlichen Repräsentation angezeigt ist, dass sie nicht im Common Ground enthalten ist und dass sie wahr ist. Der eingebettete Satz Mia ist krank in (50a) referiert auf ein Update $\sigma$, weil er F-markiert ist und durch das Matrixprädikat und das es-Korrelat angezeigt ist, dass $\sigma$ wahr ist. Die Matrixaussage $\tau$ (Max bedauert es, dass Mia krank ist) ist ebenfalls ein potenzielles Update, denn der sie repräsentierende Satz ist F-markiert und bringt durch seine deklarative ForceP den Wahrheitsanspruch von $\tau$ zum Ausdruck.

Wird bedauern wie in (54a) ohne eine es-Proform gebraucht, ist zwar durch die F-Markierung indiziert, dass $\sigma$ nicht im Common Ground enthalten ist. Dass $\sigma$ wahr ist, ist aber nicht angezeigt. Damit ist $\sigma$ kein potenzielles Update. Der Matrixsatz $\tau$ hingegen ist eins. Gleiches gilt für den Matrixsatz mit nicht-veridikalen behaupten-Prädikaten - vgl. (54b).

(54) Was gibt es Neues?

(54a) Max ${ }_{F}$ hat bedauert ${ }_{\mathrm{F}} \operatorname{pro}_{\mathrm{F}}$ [F $_{\mathrm{F}}$ dass Mia krank $\mathrm{F}_{\mathrm{F}}$ ist]]

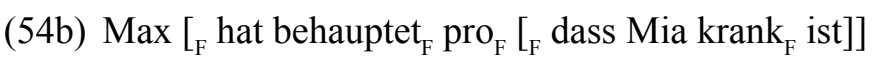

Die es-Proform indiziert also bei den potenziell faktiven bedauern-Verben und inhärent veridikalen Verben wie erreichen zusammen mit seiner F-Markierung und der des eingebetteten Satzes $\sigma$, dass letzterer als Update zu interpretieren ist. Für die Matrixaussage $\tau$ ist in diesen Fällen ebenfalls indiziert, dass es sich bei ihr um ein potenzielles Update handelt. Es gibt also im Hinblick auf (50a) zwei Updates: ,Mia ist krank' und ,Max bedauert

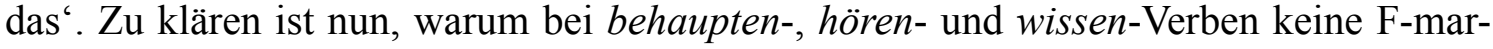
kierte es-Proform vorkommen kann - vgl. (55a-c) bzw. (50b).

(55) Was gibt es Neues?

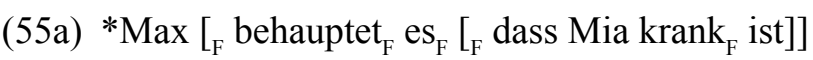

(55b) * Max [ hört $_{\mathrm{F}} \mathrm{es}_{\mathrm{F}}\left[\right.$ dass Mia krank $_{\mathrm{F}}$ ist] $]$

$(55 \mathrm{c}) * \operatorname{Max}\left[_{\mathrm{F}} \mathrm{wei}_{\mathrm{F}} \mathrm{es}_{\mathrm{F}}\left[\right.\right.$ dass Mia $_{\mathrm{F}}$ krank $_{\mathrm{F}}$ ist $\left.]\right]$

Die F-markierten es-Proformen in (55a-c) sind per definitionem nicht anaphorisch. In dem behaupten- und hören-Beispiel indiziert das Korrelat nicht, dass der eingebettete Satz ein potenzielles Update $\sigma$ bezeichnet, denn behaupten und hören werden durch das Korrelat nicht veridikal. Mit diesen Verben kann also nur die Matrixaussage als Update eingeführt werden: ,Max behauptet, dass Mia krank ist' beziehungsweise ,Max hört, dass Mia krank ist'. Damit ist das es-Korrelat funktionslos, also überflüssig. Man könnte so etwas wie ein Prinzip AVOID-es postulieren, das besagt, dass ein es-Korrelat nur dann legitim ist, wenn es anaphorisch ist oder auf ein potenzielles Update $\sigma$ verweist. ${ }^{13}$ Für (50a) und (53a-c) ist AVOID-es erfüllt, für $(55 \mathrm{a}, \mathrm{b})$ nicht.

Das es-Korrelat eines inhärent veridikalen Verbs wie wissen in (51) beziehungsweise (55c) referiert auf eine wahre Aussage $\sigma$. Wäre es F-markiert, wäre indiziert, dass $\sigma$ nicht im Common Ground enthalten ist und somit ein potenzielles Update darstellt. Dass (55c) trotzdem nicht wohlgeformt ist, liegt daran, dass ein Matrixsatz $\tau$, der einen epistemischen Zustand denotiert, keine geeignete Antwort auf eine Frage wie Was gibt es Neues? beziehungsweise kein geeignetes Update darstellt. Wenn offensichtlich ist, dass $\sigma$ wahr ist,

13 Dieses Prinzip ähnelt in gewissem Maße Chomskys (1986, S. 65) Prinzip „Avoid Pronoun“. Dieses besagt, dass in Sätzen wie John would much prefer his going to the movie die Proform his dann angemessen ist, wenn statt ihrer nicht PRO stehen kann, mit anderen Worten, wenn sie sich nicht auf John bezieht. Das heißt, wenn die Proform etwas tut, was ohne sie ohnehin der Fall ist, kann sie weggelassen werden. 
muss bei dieser Art von Fragen nicht angegeben werden, wer sich dessen bewusst ist. AVOID-es müsste somit modifiziert werden: Ein es-Korrelat ist nur dann legitim, wenn es anaphorisch ist oder wenn es auf ein Update referiert und der Matrixsatz ebenfalls. In (54a) ist dieses modifizierte AVOID-es erfüllt, in (55a-c) nicht.

Mit AVOID-es lässt sich auch erklären, warum ein es-Korrelat V2-Einbettung blockiert vgl. (30c) bzw. (56b). Der V2-Satz in (56b) ist wie jeder V2-Satz F-markiert. Damit kann das es-Korrelat nicht anaphorisch sein. Da es als Argument eines behaupten-Verbs nicht auf ein Update referiert, ist es überflüssig, verletzt also AVOID-es.

(56a) $\operatorname{Max}\left[_{F}\right.$ behauptet $\left[_{F}\right.$ Mia $\left[_{F}\right.$ ist krank]]

(56b) * Max $\left[_{F}\right.$ behauptet $\mathrm{es}_{\mathrm{F}}\left[\mathrm{F}\right.$ Mia ${ }_{\mathrm{F}}$ ist krank]]]

\subsection{Korrelate und eingebettete Interrogativsätze}

Ein eingebetteter Interrogativ denotiert eine Frage und bezieht sich dabei auf die Aussagenmenge $\{\sigma, \neg \sigma\} .{ }^{14}$ Greift ein frageeinbettendes Verb auf diese Menge zu, können in Abhängigkeit von seiner Bedeutung bestimmte Formen von Frageeinbettungen entstehen - vgl. Schwabe/Fittler (2012). Wir werden uns nun einzelne Prädikatgruppen im Hinblick darauf ansehen, welche Rolle sententiale Proformen bei der Frageeinbettung spielen.

$i$. Prädikate wie wissen, hören aber auch bedauern, die, wie wir gesehen haben, faktiv sind, wenn sie einen dass-Satz einbetten und ihre es-Proform bei sich haben, nehmen, wenn sie Fragen einbetten, Bezug auf wahre Antworten zu diesen Fragen. Sie beziehen sich dann, mit Groenendijk/Stokhof (1982) gesprochen, auf Frageextensionen. Werden sie mit einer es-Proform verwendet, referiert diese auf eine kontextgegebene wahre Antwort auf die eingebettete Frage. In (57a) referiert die es-Proform demnach entweder auf $\sigma$ oder auf $\neg \sigma$. $\mathrm{Zu}$ bemerken ist, dass (57a) äquivalent ist zu seiner korrelatlosen Entsprechung (57b).

(57a) Arnold sagt nie ein Wort. Sie kann es hören, ob sie ihn geweckt hat. (IDS rhz 2009)

(A verb es dass $\sigma \vee$ A verb es dass $\neg \sigma$ ) \& A verb es dass nicht $\sigma \Leftrightarrow \sigma$

(57b) Arnold sagt nie ein Wort. Sie kann hören, ob sie ihn geweckt hat. (A verb dass $\sigma \vee$ A verb dass $\neg \sigma$ ) \& A verb dass $\sigma \Leftrightarrow \sigma$

Anders sieht es aus, wenn zu (57a, b) die entsprechenden $w$-Formen gebildet werden.

(58a) Und die Länder wollen es dem Bund zeigen, wer das Sagen hat [...]. (DWDS Zeit 1976)

Die Länder wollen es zeigen, dass $\mu$.

(58b) Die große Gilde der Namensvettern will zeigen, wer alles zu ihnen gehört und was sie zu bieten haben. (DWDS BZ 1994)

$\forall \mathrm{x}\{[\mathrm{G}$ zeigt, dass $\mathrm{x}$ gehört_zu_G $\vee \mathrm{G}$ zeigt, dass $\mathrm{x}$ nicht gehört_zu_G] \& $[(\mathrm{G}$ zeigt, dass $\mathrm{x}$ gehört_zu_G) $\Leftrightarrow(\mathrm{x}$ gehört_zu_G) $]\}$

(58a), eine zu (57a) entsprechende $w$-Form, bezieht sich auf die kontextgegebene wahre Antwort $\mu$. Auf diese referiert die es-Proform. Bei $\mu$ kann es sich beispielsweise um ,Die Länder haben das Sagen' handeln oder um ,Ihre Ministerpräsidenten haben das Sagen'. Da nicht alle wahren Antworten in Betracht kommen, handelt es sich bei (58a) um eine nicht-exhaustive $w$-Form. In (58b) hingegen, der $w$-Entsprechung zu (57b), werden alle wahren Antworten in Betracht gezogen. Hier handelt es sich um eine exhaustive $w$-Form. Ein Korrelat ist bei letzterer ausgeschlossen.

14 Die Auffassung, dass es sich bei Fragen um Mengen von Aussagen handelt, wird u.a. von Hamblin (1987), Karttunen (1977) und Groenendijk/Stokhof (1982) vertreten. 
$W$-Formen mit evaluativen Prädikaten wie hassen und sich freuen, die übrigens keine $o b$ Fragen einbetten, beziehen sich ebenfalls auf eine wahre kontextgegebene Antwort $\mu-$ vgl. (59a, b). Kommen diese $w$-Formen mit einem Korrelat vor, referiert dieses wieder auf diese kontextgegebene wahre Antwort.

(59a) Ich liebe mein Land, aber ich hasse es, was unsere Regierung tut. (DWDS TS 2005) Ich hasse es, dass $\mu$.

(59b) Man freut sich darüber, wen das Alphabet zusammenbringt: Friedrich Nietzsche und Anais Nin, Alice Schwarzer und Albert Schweitzer. (DWDS TS 2002)

Man freut sich darüber, dass $\mu$.

ii. Wie wir in Abschnitt 3.1 gesehen haben, können Verben wie wissen und hören außer mit einem es-Korrelat auch mit einer ProPP vorkommen. Betten sie mit ihrer ProPP einen dass-Satz ein, stellt die von ihm bezeichnete Aussage eine logische Folge aus den Aussagen dar, die vom Matrixsubjekt gewusst beziehungsweise gehört werden. Beispiele mit einem eingebettetem $o b$-Satz finden sich in der Datenbank nur für hören und auch nur mit negativen Kontexten - vgl. (60).

(60) Er hatte zwar nie davon gehört, ob es in Goldmans Fall ein Gutachten gegeben hatte, aber Jeds Frau war tatsächlich die Klägerin gewesen. (gbs Fairstein 2009, isbn=3641030366)

Hier referiert der pronominale Teil der ProPP auf $\sigma$ (, In Goldmanns Fall hat es ein Gutachten gegeben'). Stellen wir uns zu (60) ein affirmatives Pendant wie (61) vor.

(61) Max hatte davon gehört, ob es in Goldmanns Fall ein Gutachten gegeben hatte.

Die ProPP in (61) referiert auf ein $\sigma$, das ein Teil einer kontextgegebenen komplexeren Formel $\varphi$ ist, für die gilt, dass Max davon gehört hat, dass $\varphi$. Ist $\varphi$ beispielsweise ,In Goldmanns Fall hat es dann ein Gutachten gegeben, wenn der Gutachter zugelassen war', hat Max davon gehört, dass es in Goldmanns Fall ein Gutachten gegeben hat, wenn der Gutachter auch zugelassen war. Die Formel $\varphi$ folgt aus dem, was Max gehört hat, muss aber nicht unbedingt von ihm gehört worden sein. Es handelt sich bei ihr um eine partielle Antwort auf die eingebettete Frage, ob es in Goldmanns Fall ein Gutachten gegeben hatte. Deutlich wird, dass im Fall von (61) anders als in den vorangegangenen Beispielen (57a, b) mit hören kein Bezug auf eine wahre Antwort auf die eingebettete Frage ausgedrückt wird. Bettet ein Matrixsatz wie (62) hingegen eine $w$-Frage ein, referiert die ProPP wie bei den Beispielen (58a) und (59a, b) auf die wahre Antwort dieser Frage.

(62) Max hatte davon gehört, von wem es ein Gutachten gegeben hatte.

iii. Betten Prädikate wie diskutieren, bedenken und nachdenken Fragen ein, nehmen sie anders als die Verben aus Gruppe (i) nicht Bezug auf die wahre Antwort der eingebetteten Frage, sondern auf die Frage selbst. Sie beziehen sich, nach Groenendijk/Stokhof (1982), auf Frageintensionen. Wie oben bemerkt, involvieren Fragen immer die Aussagenmenge $\{\sigma, \neg \sigma\}$, die sich auch als Tautologie , $\sigma \vee \neg \sigma^{6}$ darstellen lässt. Ein $o b$-Satz wie in (63a, b) bezieht sich auf so eine Tautologie. Ist wie in (63a', b') ein Korrelat gegeben, so referiert dieses auf die Tautologie, hat aber kaum Einfluss auf die Interpretation des Matrixprädikats und des eingebetteten Satzes. ${ }^{15}$

(63a) Als die Algerier mich um politische Hilfe baten, habe ich lange nachgedacht, ob ich das gegenüber Frankreich verantworten kann. (DWDS BZ 2004) 15 Schwabe/Fittler (2012) nehmen an, dass durch die Korrelate die Gültigkeit des $o b$-Prädikats einge-
schränkt wird auf Aussagen, die weder tautologisch noch kontradidaktisch sind. 
(63a') Erst mit 30 werde ich darüber nachdenken, ob ein Ende meiner Karriere in Sicht kommt, meinte der sympathische Schwede, [...]. (DWDS BZ 1994)

(63b) Eine Weile haben wir überlegt, ob wir die Diplomaten mit einer Kapelle und der „Berliner Luft empfangen sollten“, [...]. (DWDS BZ 1994)

(63b')Und selbst ein Ministerpräsident wird es sich jetzt ganz genau überlegen, ob er sich mit Merkel anlegen will. (DWDS BZ 2005)

Betten Verben wie diskutieren, bedenken und nachdenken einen $w$-Satz ein, kann sich dieser ebenfalls auf eine Tautologie beziehen, auf $\mu \vee \neg \mu$. Bei $\mu$ handelt es sich wieder um eine kontextgegebene Aussage. Das Korrelat referiert dann ähnlich wie bei (63a', b') auf eine Tautologie - vgl. (64a, b).

(64a) Was würden Sie sich wünschen, was die Besucher aus dem Film mitnehmen? Daß sie noch mal genau darüber nachdenken, wen sie bei der nächsten Bundestagswahl wählen. (DWDS BZ 1998)

(64b) In diesen Momenten fängt er an nachzudenken, was ihn eigentlich noch hält an diesem Leben. (DWDS BZ 2005)

Es ist aber auch möglich, dass das Korrelat auf die wahre Antwort auf die eingebettete Frage referiert.

(65) Was würden Sie als Erstes tun? Ich würde darüber nachdenken, wer mich gewählt hat und was diese Wähler wollen. (DWDS BZ 2003)

Eine $w$-Form mit einem Verb wie nachdenken kann sich also entweder auf eine Frageextension oder auf eine Frageintension beziehen. Die Interpretation hängt bei den Beispielen $(64 a, b)$ und (65) vom Kontext ab. Ist kein Korrelat gegeben, wie in (64b), denotiert die Frage eine Frageintension. Mit Korrelat ist es entweder die Frageintension (64a) oder die Frageextension (65).

Der Abschnitt zu Frageeinbettung und Korrelaten hat gezeigt, dass Korrelate auf kontextgegebene Aussagen referieren. Handelt es sich bei der Aussage um eine kontextgegebene Antwort $\mu$ auf eine durch das Matrixprädikat eingebettete $w$-Frage, muss sie im Common Ground enthalten sein. Das heißt, ein Korrelat mit einem $w$-Satz als Bezugssatz ist immer anaphorisch. Anders sieht es bei Korrelaten aus, die einen ob- oder dass-Satz als Bezugssatz haben. Sie können, wie wir in (12a) beziehungsweise (50a) und (26) gesehen haben, auf eine Aussage verweisen, die nicht im Common Ground enthalten ist.

\section{Zusammenfassung}

In diesem Aufsatz wurden Korrelate generell als Proformen angesehen, die jeweils auf eine Aussage referieren. Gegen die Homonymieansätze von Sudhoff (2003), Frey (2011) und Zimmermann (i.Vorb.), nach denen es sich bei es-Korrelaten bei behaupten-Verben stets um „anaphorische Proformen“ mit rechtsversetztem Bezugssatz handelt und bei bedauern-Verben entweder um „Platzhalter" mit extraponiertem Bezugssatz oder um anaphorische Proformen, wurden folgende Kritikpunkte angeführt: $i$. Bei behaupten-Verben kann der Bezugssatz mit dem es-Korrelat im Verbkomplex topikalisiert werden (21), was gegen die Rechtsversetzung des Bezugssatzes spricht. ii. Außer ansatzweise bei Sudhoff (2003) wird nicht gezeigt, welche Funktion das Platzhalter-es als „Determinierer“ hat. So geht aus Sternefeld (2006) nicht hervor, warum das Platzhalter-es overt (15b) oder nicht overt (17a) sein kann. Und Müller (1995), Frey (2011) und Zimmermann (i.Vorb.) zeigen 
nicht, warum es bei gleichem Matrixprädikat eingebettete CPn mit oder ohne Determinierer gibt - vgl. (15b) und (19c). iii. Ein F-markiertes es-Korrelat bei ergänzenden wennSätzen kann nur eine Proform sein, nicht aber ein so genanntes Platzhalter-es - vgl. (24) und (25). $i v$. Es wird nicht in Betracht gezogen, welchen Status Korrelate bei der Frageeinbettung haben - vgl. (26) und Abschnitt 3.3.

Bei den von Frey (2011) angeführten empirisch basierten Argumenten für einen dritten Korrelattyp, das thematische es, wurden seine Argumente als nicht zwingend für die Annahme dieses dritten Typs angesehen.

Die hier propagierte Uniformitätsanalyse sieht das jeweilige Korrelat als basisgeneriertes syntaktisches Komplement und den Bezugssatz als basisgeneriertes VP-Adjunkt an. Für korrelatlose Strukturen wurde ein pro in der Komplementposition angenommen, das seinen Bezugssatz m $\mathrm{m}_{\mathrm{e}}$-kommandieren muss - vgl. (33a-c), (39) und (44a). Mit der Uniformitätsanalyse ließ sich erklären, dass der Bezugssatz bei behaupten-Verben innerhalb des korrelathaltigen Verbkomplexes topikalisiert werden kann (21) und dass das Subjekt-es bei der Topikalisierung des Verbalkomplexes obligatorisch ist (39a). Letzteres wurde damit erklärt, dass ein pro in der Subjektposition nur dann legitim ist, wenn es seinen Bezugssatz $\mathrm{m}_{\mathrm{e}}$-kommandiert - vgl. (41). Des Weiteren hat es sich ergeben, dass F-markierte Korrelate in ergänzenden wenn-Formen und $o b$-Formen unproblematisch sind vgl. (24) und (26).

Es wurde dafür argumentiert, dass ein Korrelat als sententiale Proform auf eine abstrakte Aussage referiert, die direkt durch den Bezugssatz bezeichnet wird, wenn es sich bei diesem um einen dass- oder wenn-Satz handelt. Die Aussage wird durch den Bezugssatz indirekt bezeichnet, wenn es sich bei ihm um eine Frage handelt. Eine nicht-anaphorische es-Proform zu einem eingebetteten dass-Satz ist dann legitim, wenn sie F-markiert ist und zusammen mit dem Matrixprädikat die eingebettete Aussage als wahr kennzeichnet. Ist das der Fall, denotiert der eingebettete Satz ein potenzielles Update - vgl. die F-Markierung der es-Proform und die Faktivitätsinduzierung bei bedauern-Verben in (54a). Dass behaupten-Verben F-markierte es-Proformen verbieten, wurde damit erklärt, dass das esKorrelat überflüssig ist, wenn es zusammen mit dem Matrixprädikat eine Aussage nicht als wahr kennzeichnet. Bei Frageeinbettungen referieren Korrelate verb- und korrelattypabhängig auf kontextgegebene vollständige oder partielle (wahre) Antworten auf Fragen oder auf Tautologien.

\section{Verwendete Abkürzungen in den Quellenangaben}

\begin{tabular}{|c|c|}
\hline brz & Braunschweiger Zeitung \\
\hline $\mathrm{BZ}$ & Berliner Zeitung \\
\hline dpr & Die Presse \\
\hline DWDS & $\begin{array}{l}\text { Digitales Wörterbuch der deutschen Sprache der Berlin-Brandenburgischen Akademie der } \\
\text { Wissenschaften }\end{array}$ \\
\hline gbs & Google Buchsuche \\
\hline IDS & Deutsches Referenzkorpus des Instituts für Deutsche Sprache in Mannheim \\
\hline $\mathrm{K}-\mathrm{Ze}$ & Kernkorpus-Zeitungen \\
\hline rhz & Rheinische Zeitung \\
\hline & Tagesspiegel (Berlin) \\
\hline eit & Die Zeit \\
\hline
\end{tabular}




\section{Literatur}

Asher, Nicholas (1993): Reference to abstract objects in discourse. Dordrecht: Kluwer. (= Studies in Linguistics and Philosopy 50).

Axel-Tober, Katrin (2012): (Nicht-)kanonische Nebensätze im Deutschen: synchrone und diachrone Aspekte. Berlin u.a.: de Gruyter. (= Linguistische Arbeiten 542).

Bennis, Hans (2004): Unergative Adjectives and Psych Verbs. In: Alexiadou, Artemis/Anagnostopoulou, Elena/Everaert, Martin (Hg.): Studies in Unaccusativity: The Syntax-Lexicon Interface. Cambridge: CUP. S. 84-113. (= Oxford Studies in Theoretical Linguistics 5).

Berman, Judith (2003): Clausal Syntax of German. Stanford, CA: CSLI. (= Studies in Constraint-Based Lexicalism).

Breindl, Eva (1989): Präpositionalobjekte und Präpositionalobjektsätze im Deutschen. Berlin: Niemeyer. (= Linguistische Arbeiten 220).

Chomsky, Noam (1986): Lectures on Government and Binding. Dordrecht: Foris Publications. (= Studies in Generative Grammar 9).

Cinque, Guglielmo (1990): Ergative Adjectives and the Lexicalist Hypothesis. In: Natural Language and Linguistic Theory 8, S. 1-39.

Égré, Paul (2008): Question-Embedding and Factivity. In: Grazer Philosophische Studien 77, S. 85-125.

Fabricius-Hansen, Catherine (1980): Sogenannte ergänzende wenn-Sätze. Ein Beispiel syntaktisch-semantischer Argumentation. In: Kopenhagener Beiträge zur germanistischen Linguistik, Sonderband 1. Kopenhagen.

Frey, Werner (2011): On the 'nominal' character of clauses associated with a pronominal. Vortrag auf der 33. Jahrestagung der DGfS, Göttingen, 23.02-25.02. AG 11. Internet: www.zas.gwz-berlin.de/fileadmin/ veranstaltung_zas/workshops/archiv/proforms/frey-pronominal.pdf.

Groenendijk, Jeroen/Stokhof, Martin (1982): Semantic Analysis of WH-Complements. In: Linguistics and Philosophy 5, S. 175-233.

Haider, Hubert (2010): The Syntax of German. Cambridge: CUP. (= Cambridge Syntax Guides).

Hamblin, C. Leonard (1973): Questions in Montague English. In: Foundations of Language 10, S. 41-53.

Hintikka, Jaakko (1976): The Semantics of Questions and the Questions of Semantics. In: Acta Philosophica Fennica 28, S. 4.

Huddleston, Rodney/Pullum, Geoffrey K. (2002): The Cambridge Grammar of the English Language. Cambridge: CUP.

Karttunen, Lauri (1977): Syntax and semantics of questions. In: Linguistics and Philosophy 1, S. 3-44.

Kaiaty, Mohamed (2010): Überlegungen zu sog. 'ergänzenden wenn-Sätzen' im Deutschen. In: Deutsche Sprache 38, S. 287-308.

Meinunger, André (2007): About object es in the German ,Vorfeld‘. In: Linguistic Inquiry 38, S. 553-563.

Müller, Gereon (1995): On extraposition \& successive cyclicity. In: Lutz, Uli/Pafel, Jürgen (Hg.): On Extraction and Extraposition in German. Amsterdam/Philadelphia: Benjamins. S. 213-243. (= Linguistik aktuell 11).

Müller, Gereon/Sternefeld, Wolfgang (1993): Improper Movement and Unambiguous Binding. In: Linguistic Inquiry 24, S. 461-507.

Pesetsky, David (1991): Zero Syntax, Part II. Unver. Ms., MIT. [http://web. mit.edu/linguistics/people/ faculty/pesetsky/publications.html]

Pütz, Herbert (1986): Über die Syntax der Pronominalform es im modernen Deutsch. Tübingen: Narr. (= Studien zur deutschen Grammatik 3). 
Reis, Marga (1997): Zum syntaktischen Status unselbständiger Verbzweit-Sätze. In: Dürscheid, Christa/ Ramers, Karl Heinz/Schwarz, Monika (Hg.): Sprache im Fokus. Tübingen: Niemeyer. S. 121-144.

Schwabe, Kerstin (i.Vorb.): Sentential proforms and argument conditionals. In: Frey, Werner/Meinunger, André/Schwabe, Kerstin (Hg.): Inner-sentential propositional pro-forms: syntactic properties and interpretative effects. Amsterdam/Philadelphia: Benjamins. (= Linguistik aktuell).

Schwabe, Kerstin (2012): The German Sentential Proform ,es“ in All-Focus Sentences. In: Brandtler Johan et al. (Hg.): Discourse \& Grammar (A Festschrift in Honor of Valéria Molnár). Lund: Lund University. S. 459-474.

Schwabe, Kerstin/Fittler, Robert (2009): Semantic characterizations of German question-embedding predicates. In: Bosch, Peter/Gabelaia, David/Lang, Jérôme (Hg.): Logic, language, and computation: 7th international Tbilisi symposium on logic, language, and computation, TbiLLC 2007, Tbilisi, Georgia, October 1-5, 2007, revised selected papers. Berlin: Springer. S. 229-241. (= Lectures Notes in Artificial Intelligence 5422).

Schwabe, Kerstin/Fittler, Robert (2012) Über semantische Konsistenzbedingungen deutscher Matrixprädikate. Ms. ZAS Berlin.

Schwarzschild, Roger (1999): Givenness, AvoidF and other constraints on the placement of accent. In: Natural Language Semantics 7(2), S. 141-177.

Stalnaker, Robert (2002): Common Ground. In: Linguistics and Philosophy 25, S. 701-721.

Sternefeld, Wolfang (2006): Syntax. Eine morphologisch motivierte generative Beschreibung des Deutschen. Bd. 1. Tübingen: Stauffenburg. (= Stauffenburg-Linguistik 31.1).

Sudhoff, Stefan (2003): Argumentsätze und es-Korrelate - zur syntaktischen Struktur von Nebensatzeinbettungen im Deutschen. Berlin: Wissenschaftlicher Verlag.

Sudhoff, Stefan (i.Vorb.): Correlates of object clauses in German and Dutch. In: Frey, Werner/Meinunger, André/Schwabe, Kerstin (Hg.): Inner-sentential propositional pro-forms: syntactic properties and interpretative effects. Amsterdam/Philadelphia: Benjamins. (= Linguistik aktuell).

Trompelt, Helena/Axel-Tober, Katrin/Holler, Anke (i.Vorb.): Correlative Es vs. Das in German: An Experimental Perspective. In: Frey, Werner/Meinunger, André/Schwabe, Kerstin (Hg.): Inner-sentential propositional pro-forms: syntactic properties and interpretative effects. Amsterdam/Philadelphia: Benjamins. (= Linguistik aktuell).

Zifonun, Gisela/Hoffmann, Ludger/Strecker, Bruno (1997): Grammatik der Deutschen Sprache. Bd. II. Berlin/New York: de Gruyter. (= Schriften des Instituts für deutsche Sprache 7.2).

Zimmermann, Ilse (i.Vorb.): Phonological, morphosyntactic and semantic properties of es In: Frey, Werner/ Meinunger, André/Schwabe, Kerstin (Hg.): Inner-sentential propositional pro-forms: syntactic properties and interpretative effects. Amsterdam/Philadelphia: Benjamins. (= Linguistik aktuell).

Kerstin Schwabe

Zentrum für Allgemeine Sprachwissenschaft

Schützenstrasse 18

D-10117 Berlin

E-Mail: schwabe@zas.gwz-berlin.de 